\title{
Abundance gradients in elliptical galaxies
}

\author{
L. Angeletti and P. Giannone
}

\author{
Dipartimento di Fisica, Università "La Sapienza”, Piazzale A. Moro, 500185 Roma, Italy \\ e-mail: lucio.angeletti@uniroma1.it;pietro.giannone@uniroma1.it
}

Received 27 March 2002 / Accepted 30 January 2003

\begin{abstract}
Abundance gradients predicted by dissipative models of galaxy formation are studied with the aid of mass models of spherical galaxies obeying the Sersic $R^{1 / n}$ law in projection. The link between metallicity and stellar binding energies and angular momenta is derived from the "concentration model" by Lynden-Bell (1975) with the addition of a terminal wind and an age spread among the stellar populations of individual ellipticals. By using the calibration of the $M g_{2}$ index as a function of metallicity and age from Single-burst Stellar Populations models, the radial profiles of $M g_{2}$ are computed for a variety of models to illustrate the general predictions of the present approach. We also matched the observed radial profiles of $M g_{2}$ in a sample of 11 ellipticals. We find that observations are reproduced reasonably well in our adopted approximation and require a considerable degree of dissipation and a metal yield above the solar value. A terminal wind or an age spread make it easier to fit the $M g_{2}$ profiles of the ellipticals with shallow $M g_{2}$ profiles in the innermost regions. However, model results (with or without an age spread) show that the exact determination of the free parameters suffers from degeneracy of their effects. Moreover, model results also suffer from the poorly known effects of non-solar abundance ratios on index $M g_{2}$. Other spectral indices, besides $M g_{2}$, and broad-band colours observed in elliptical galaxies can be examined in the same way with the present scheme; their study is postponed to a further investigation.
\end{abstract}

Key words. galaxies: elliptical and lenticular

\section{Introduction}

Colour and line-strength gradients observed across elliptical galaxies (Es) are consistent with the hypothesis that such gradients stem from abundance gradients that developed when Es formed (Franx et al. 1989; Peletier et al. 1990; Davies et al. 1993; Carollo et al. 1993).

Two basic scenarios have been proposed so far for the formation of elliptical galaxies. According to the conventional view, all luminous ellipticals are coeval and old systems (Visvanathan \& Sandage 1977; Bower et al. 1992) that formed through a monolithic dissipative collapse (Eggen et al. 1962; Larson 1974; Arimoto \& Yoshii 1987), occurred early in the evolution of the Universe and completed at an epoch much smaller than the Hubble time. The alternative scenario proposes that the ellipticals formed through a lengthy hierarchical clustering of small objects into larger ones with an extended star formation over time (Cole et al. 1994; Baugh et al. 1996; Kauffmann 1996; Kauffmann \& Charlot 1998).

Metallicity gradients in Es are predicted generally by dissipative models of galaxy formation (Larson 1976; Carlberg 1984; Theis et al. 1992). An alternative explanation (Franx \& Illingworth 1990) suggests that, across an E galaxy, the star formation proceeds near the centre for a time longer than farther

Send offprint requests to: L. Angeletti, e-mail: lucio. angeletti@uniroma1.it out, according to the local escape velocity (see also Martinelli 1998). However, the behaviour of observed indices varies so widely from one galaxy to another that it is not clear whether the explanations mentioned above can account for all individual differences (Pagel 1997).

In this paper we follow the conventional view by adopting the dissipative model of galaxy formation in the simplified analytical version by Lynden-Bell (1975, hereafter LB). The "Concentration Model" (hereafter CM) by LB provides an analytical formula for the star-metallicity distribution, related explicitly to the degree of dissipation and to the metal yield, thus making it easier to explore the corresponding two-parameter space.

In adopting the conventional view, we have to consider the evidence found by various authors (González 1993; Tantalo et al. 1998; Jørgensen 1999; Kuntschner 2000; Trager et al. 2000a,b; Vazdekis et al. 2001; Terlevich \& Forbes 2002) of an age spread among the ellipticals. In particular, the central regions of most Fornax and Virgo ellipticals seem old with an age spread of a few Gyr (Kuntschner 2000; see also Trager et al. 2000a,b, hereafter TFWG1 and TFWG2, respectively), whereas the innermost regions of the field or ellipticals in small groups show a wide age spread (from 1.5 to $18 \mathrm{Gyr}$ ), suggesting an environmental effect (TFWG1-2). Furthermore, age gradients are also found within individual ellipticals (TFWG1), with ages getting older by $25 \%$ on the average, when going from the 
regions within $R_{\mathrm{e}} / 8$ ( $R_{\mathrm{e}}$ being the effective radius) to the outer regions within $R_{\mathrm{e}} / 2$.

We follow TFWG2, who favour a variant of the conventional view by suggesting a minor contamination of the base old population in ellipticals by younger stars (see also Kodama \& Arimoto 1997, 1998) that formed from well-mixed preenriched gas rather than unmixed low-metallicity gas from an accreting object. The CM, as formulated by $\mathrm{LB}$, is compatible with an age spread among ellipticals, but it does not take into account the age spread among the stellar populations, that formed within each elliptical galaxy successively. We shall show that such an age dependence, together with the suggestion by TFWG2, can be added easily to the analytical formulation of the CM.

The CM is used here together with the spherical $R^{1 / 4}$-mass model of elliptical galaxies derived, by deprojection (Young 1976), from the surface-brightness profile obeying the $R^{1 / 4}$ law (de Vaucouleurs 1948). However, we shall also consider some cases of the $R^{1 / n}$-mass models ( $n=2$ to 8 ) obtained by deprojecting (Ciotti 1991) the generalized $R^{1 / n}$ law (Sersic 1968). Minor modifications to CM, as formulated by LB, are introduced to include a terminal wind and an age spread, and to adapt $\mathrm{CM}$ to the $R^{1 / n}$ model. In this paper we describe our approach and, as a first application to elliptical galaxies, we present the computation of the integrated absorption-line index $\mathrm{Mg}_{2}$ and a comparison of the model results with the observed radial profiles of $M g_{2}$ in 11 elliptical galaxies.

The paper is arranged as follows: Sect. 2, after reviewing the basic assumptions of CM, describes its adjustment to the $R^{1 / n}$ model and the inclusion of a terminal wind and an age spread; Sect. 3 presents the data for Single-burst Stellar Populations used in the paper, and the model predictions concerning the $\mathrm{Mg}_{2}$ index; Sect. 4 compares the model results to the $M g_{2}$ radial profiles in a sample of 11 elliptical galaxies; Sect. 5 gives a final discussion with our conclusions. The derivation of the metallicity-distribution function is given in Appendix A for the general case and in Appendix B for some specific cases of CM with a terminal wind. Technical details on how the CM is adjusted to the $R^{1 / n}$ model are assembled in Appendix C.

\section{The Concentration Model}

In this section we first review the basic assumptions of CM, succinctly referred to in LB, and then present some minor adjustments required by the $R^{1 / n}$ model, the introduction of a terminal wind, and the consideration of an age spread among the stellar populations.

\subsection{Basic assumptions}

We limit ourselves to a spherical galaxy of total mass $M_{0}$ at the initial time $t_{0}$, and consisting of gas with uniform metallicity $Z_{0}$. We denote by $t_{\mathrm{w}}$ the final epoch when the star formation ceases, and by $G(t)$ the gas mass at the epoch $t$ intermediate between $t_{0}$ and $t_{\mathrm{w}}$. Setting $G_{\mathrm{w}}=G\left(t_{\mathrm{w}}\right)$, the final total mass of the stellar component (long-living stars and compact stellar remnants) is $M_{\mathrm{s}}=M_{0}-G_{\mathrm{w}}$.
The mass distribution of the stellar component, in a gasfree and steady-state galaxy after $t_{\mathrm{w}}$, is referred to a Lagrangian mass coordinate $m$, in units of $M_{\mathrm{s}}$ and varying from 0 at the centre to 1 in the outermost zones. Metallicities of stars, in e.g. the mass shell $m$ to $m+\mathrm{d} m$, are inferred by going back to the time, before $t_{\mathrm{w}}$, when those stars formed. Therefore, the $\mathrm{CM}$ describes the mass distribution of the stellar component at an epoch before $t_{\mathrm{w}}$ by using the same mass coordinate $m$ as adopted after $t_{\mathrm{w}}$. It is assumed that spherical surfaces (onionwise about one another) corresponding to constant values of $m$ were associated with the first-generation stars that formed on them and were slowly moving with the long-term average motions of such stars. Here we consider the case $G_{\mathrm{w}}=0$ so that $M_{\mathrm{s}}=M_{0}$ (as in LB), deferring the case $G_{\mathrm{w}}>0$ to Appendix A.

The gas is assumed to be well-mixed and its uniform metallicity $Z$ is evaluated according to the Simple Model (Pagel \& Patchett 1975) as

$$
Z=Z_{0}-p \ln g
$$

where $p$ is the metal yield and $g=G(t) / M_{0}$. Conversely, one has $g \equiv g(Z)=\exp \left[-\left(Z-Z_{0}\right) / p\right]$. In the following, we shall use Eq. (1), although it holds only for $Z<<1$, and should be replaced by $Z=1-\left(1-Z_{0}\right) g^{p}$ when $Z$ approaches 1 . As a result of the gas consumption by the star formation and of the energy dissipation by the cloud-cloud collisions, the gas contracts gradually with respect to the stars and is confined, at the time $t_{\mathrm{a}}$, within the decreasing mass coordinate $m_{\mathrm{a}}$ given by the ansatz

$m_{\mathrm{a}}=g_{\mathrm{a}}^{c}$

where $g_{\mathrm{a}} \equiv g\left(t_{\mathrm{a}}\right)$ and $0<c<1$ ( $c$ being the concentration index); the corresponding gas metallicity is $Z_{\mathrm{a}}=Z_{0}-(p / c) \ln m_{\mathrm{a}}$. The special function $g_{\mathrm{a}}^{c}$ ensures that " $m_{\mathrm{a}}-g_{\mathrm{a}}$ " (the mass of the stars within $m_{\mathrm{a}}$ ) is zero for $g_{\mathrm{a}}=1$ and $g_{\mathrm{a}}=0$, and non-negative for $0<g_{\mathrm{a}}<1$, as it must be.

For the stars formed inside $m_{\mathrm{a}}$, the CM makes the plausible assumptions that: i) the star formation is distributed radially like the stars born before $t_{\mathrm{a}}$ (Appendix A), ii) the stars that form at $t_{\mathrm{a}}$, from inelastic collisions between clouds confined within $m_{\mathrm{a}}$, have the orbital apocentres within $m_{\mathrm{a}}$. From item ii) one gets that the mass of the stars within $m$, having metallicities not larger than $Z_{\mathrm{a}}$, and born until $t_{\mathrm{a}}$, is

$s\left(m, Z_{\mathrm{a}}\right)=m-g_{\mathrm{a}}$

where $m \geq m_{\mathrm{a}}$ and $g_{\mathrm{a}}=g\left(Z_{\mathrm{a}}\right)$.

\subsection{The modified Concentration Model}

Let us now consider the final distribution of the stellar component. The stars with apocentres inside $m_{\mathrm{a}}$ (partially formed at the time $t_{\mathrm{a}}$ ) are all confined within the sphere $m_{\mathrm{a}}$ and their cumulative mass will be denoted by $s_{1}\left(m_{\mathrm{a}}\right)$. The stars with apocentres outside $m_{\mathrm{a}}$ (completely formed at the time $t_{\mathrm{a}}$ ) are found within $m_{\mathrm{a}}$ and outside $m_{\mathrm{a}}$ and their cumulative masses will be indicated by $s_{2}\left(m_{\mathrm{a}}\right)=m_{\mathrm{a}}-s_{1}\left(m_{\mathrm{a}}\right)$ and $s_{3}\left(m_{\mathrm{a}}\right)=1-m_{\mathrm{a}}$, respectively. 

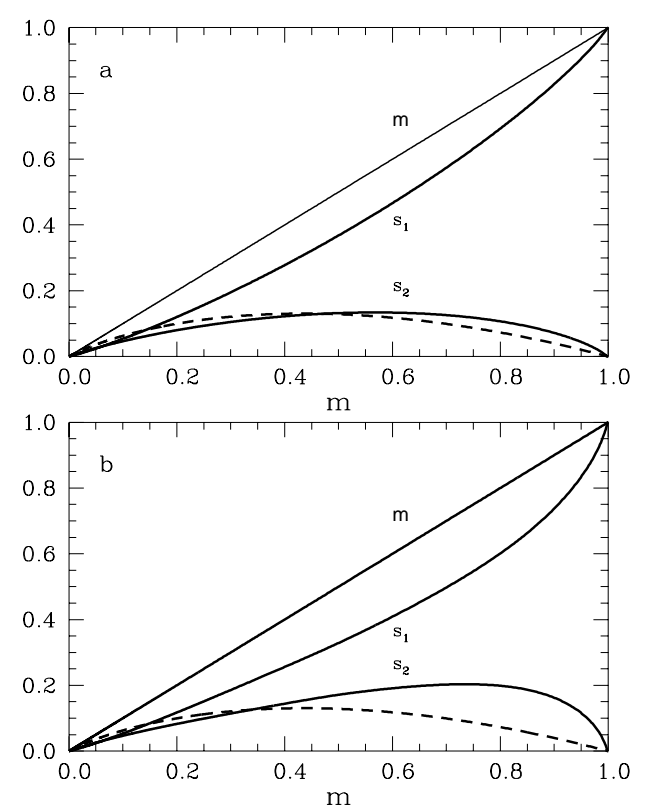

Fig. 1. a) Functions $s_{1}(m)$ and $s_{2}(m)$, of the isotropic $R^{1 / 4}$ model, versus the mass coordinate $m$, and function $m-m^{1 / c}$ (for the case $c=0.7$, dashed curve), see text. b) As in panel a) but for the anisotropic $R^{1 / 4}$ model with anisotropy radius $r_{\mathrm{a}}=1$.

Since $s\left(m_{\mathrm{a}}, Z_{\mathrm{a}}\right)$ contains mass $s_{2}\left(m_{\mathrm{a}}\right)$, from Eq. (3) one should have $m_{\mathrm{a}}-g_{\mathrm{a}}=m_{\mathrm{a}}-m_{\mathrm{a}}^{1 / c} \geq s_{2}\left(m_{\mathrm{a}}\right)$ : an inequality not unconditionally true, e.g. for the $R^{1 / n}$ model (see Fig. 1 for the case $n=4)$. To satisfy the condition $s\left(m_{\mathrm{a}}, Z_{\mathrm{a}}\right)-s_{2}\left(m_{\mathrm{a}}\right)=$ $s_{1}\left(m_{\mathrm{a}}\right)-g_{\mathrm{a}} \geq 0$, one can define $m_{\mathrm{a}}$ implicitly by the ansatz

$s_{1}\left(m_{\mathrm{a}}\right)=g_{\mathrm{a}}^{c}$

and then introduce quantity $\sigma\left(m, Z_{\mathrm{a}}\right)=s\left(m, Z_{\mathrm{a}}\right)-s_{2}(m)$, as the mass of the stars with apocentres inside $m$, born up to $t_{\mathrm{a}}$, and with metallicities not larger than $Z_{\mathrm{a}}=Z_{0}-(p / c) \ln s_{1}\left(m_{\mathrm{a}}\right)$. The star formation within $m_{\mathrm{a}}$ is now assumed to be radially distributed like the stars with apocentres inside $m_{\mathrm{a}}$ and formed up to $t_{\mathrm{a}}$. Note that Eq. (4) exploits the property $s_{1}(0)=0$ and $s_{1}(1)=1$ of the monotonic function $s_{1}\left(m_{\mathrm{a}}\right)$ (see Fig. 1).

In order to include a terminal wind, which removes the gas mass $g_{\mathrm{w}}=G_{\mathrm{w}} / M_{0}$ from the sphere $m_{\mathrm{w}} \leq 1$, we expand Eq. (4) into the ansatz

$s_{1}\left(m_{\mathrm{a}}\right)=s_{1}\left(m_{\mathrm{w}}\right)+\left[1-s_{1}\left(m_{\mathrm{w}}\right)\right]\left(\frac{g_{\mathrm{a}}-g_{\mathrm{w}}}{1-g_{\mathrm{w}}}\right)^{c}$

which gives $m_{\mathrm{a}}$ in the range 1 to $m_{\mathrm{w}}$, for $g_{\mathrm{a}}$ varying from 1 to $g_{\mathrm{w}}$, and reduces to (4) for $m_{\mathrm{w}}=g_{\mathrm{w}}=0$.

The cumulative mass $\sigma\left(m, Z_{\mathrm{a}}\right)$ and the inferred metallicitydistribution function $M D F$, both ensuing from Eq. (5), are as in LB for $m_{\mathrm{w}}=0$; their derivation for $m_{\mathrm{w}}>0$ is given in Appendix A. The analytical expression of $\sigma\left(m, Z_{\mathrm{a}}\right)$ is explicitly given in Appendix B for some cases of CM with a terminal wind as considered in Sect. 3.3. Finally, Appendix C explains how function $M D F$ is adapted to the $R^{1 / n}$-mass model by transferring the dependence of $M D F$ on the orbital apocentre and mass coordinate $m$ into a dependence on the binding energy and angular momentum.

\subsection{The age of stellar populations}

The CM implies that the stellar populations within an elliptical have ages $\tau=t_{\text {now }}-t$ (where $t_{\text {now }}$ corresponds to the present epoch) ranging from the initial age $\tau_{0}=t_{\text {now }}-t_{0}$ to the final age $\tau_{\mathrm{w}}=t_{\text {now }}-t_{\mathrm{w}}$. The age dependence can be added to the CM by combining the Simple Model with the one-zone approach of chemical evolution (Pagel 1997) and the Schmidt's law (Schmidt 1959) for the star formation rate (Pagel \& Patchett 1975), thus obtaining

$\frac{\mathrm{d} g}{\mathrm{~d} t}=-v g^{k} \quad($ for $\quad 1 \leq k \leq 2)$

where $t_{0} \leq t \leq t_{\mathrm{w}}$ with $g\left(t_{0}\right)=1$ and $v^{-1}$ being the characteristic time of the gas conversion into stars. By adopting $k=1$, one gets

$g(t)=\exp \left[-v\left(t-t_{0}\right)\right]$

Equation (7) provides the relation

$\tau=\tau_{0}+\frac{\ln g}{v}$

between the age $\tau$ and $g$, and, by writing $g=\exp \left[-\left(Z-Z_{0}\right) / p\right]$ from Eq. (1), the relation

$\tau=\tau_{0}-\frac{\left(Z-Z_{0}\right)}{p v}$

between $\tau$ and $Z$. For fixed $\tau_{0}$ and $\tau_{\mathrm{w}}$, Eq. (8) gives $v$ by imposing $g=g_{\mathrm{w}}$ at $\tau=\tau_{\mathrm{w}}$. Furthermore, to adapt the CM to the suggestions by TFWG2 and Kodama \& Arimoto (1997 and 1998), referred to in Sect. 1, one can assume a main episode of star formation, occurring until $t=t_{1}$ and involving the gas mass $1-g\left(t_{1}\right)$, and a delayed minor episode occurring from $t_{2}\left(>t_{1}\right)$ to $t_{\mathrm{w}}$ and involving the gas mass $g\left(t_{1}\right)-g_{\mathrm{w}}$.

An obvious improvement of our procedure would be the one-zone model developed by various authors (Arimoto \& Yoshii 1987; Matteucci \& Tornambé 1987; Bressan et al. 1994; Tantalo et al. 1996; Vazdekis et al. 1996) where the instantaneous recycling approximation of the Simple Model is replaced by realistic stellar lifetimes, or the hydrodynamical approach (Larson 1976; Theis et al. 1992) where the one-zone approximation is superseded by a star-formation rate that depends closely on the local gas density.

\section{The integrated index $M g_{2}$}

To see whether abundance gradients observed in ellipticals can be matched by the metallicity gradients predicted by the Concentration Model, we employ the spectral index $M g_{2}$ of the Lick/IDS system (Burstein et al. 1984; Worthey et al. 1994; Trager et al. 1998), since it is one of the most widely used of the Lick indices and a large body of accurate observations is available (see Trager et al. 1998 and references therein). In the following we describe our procedure to compute the radial profiles of $\mathrm{Mg}_{2}$, for various choices of the concentration index $c$ and the metal yield $p$, and compare them to observations. The initial abundance $Z_{0}=0$ and the solar metallicity $Z_{\odot}=0.0169$ are adopted throughout the paper. 


\subsection{The spectral index $\mathrm{Mg}_{2}$}

$M g_{2}$ is the deficit of flux, expressed in magnitudes at the $\mathrm{Mgb}+\mathrm{MgH}$ feature, compared with a nominal continuum (pseudocontinuum) determined by interpolation from neighbouring regions. $M g_{2}$ is very sensitive to the $\mathrm{Mg}$ abundance, but also depends on several other chemical species (see Tripicco \& Bell 1995). We shall refer to the index $M g_{2}$ for a Single-burst Stellar Population (SSP) of given $Z$ and age $\tau$, which is derived from various sources, as described e.g. in Worthey (1994; see also Charlot et al. 1996).

The SSP index $M g_{2}$ is used here to compute the "integrated" index $M g_{2}$ for the stars that are seen in projection along a line-of-sight or within a circular aperture. Such stars span a range in metallicity $Z$ and age $\tau$ as predicted by the CM and the $R^{1 / n}$-mass model. Our integrated index $M g_{2}$ is then compared to the $M g_{2}$ observed in ellipticals. Technical details on the computation of the integrated index $\mathrm{Mg}_{2}$ from availaible SSP data are given in Sect. 3.2 and in Appendix C.

\subsection{Sources of SSP data}

We need SSP libraries providing $M g_{2}$ and luminosities $L_{B}$ and $L_{V}$ in the $B$ and $V$ photometric bands, respectively, at a fair grid of $Z$ values ranging from $Z \simeq 0$ up to at least $Z=0.05$ and possibly $Z \simeq 0.1$, in order to make interpolations and/or extrapolations as smooth and reliable as possible. We found three such libraries: i) Bressan et al. (1996, hereafter BCT) and Tantalo et al. (1996), ii) Buzzoni et al. (1992, hereafter BGM) and Buzzoni (1989 and 1995), and iii) Worthey (1994, hereafter W94). The data from BGM include a red-horizontal-branch morphology and a mass-loss parameter equal to 0.3 . The behaviour of index $\mathrm{Mg}_{2}$ from BCT, BGM, and W94 is shown in Fig. 2.

The mentioned datasets use 6 or 7 SSP metallicities from $Z \simeq 0.0001$ up to $Z=0.0534$ (W94) or 0.1 (BCT and BGM), and adopt a power-law initial mass function (IMF) with the mass exponent $x=2.35$ (Salpeter's value). Furthermore, the data we derive from them refer to the "luminous mass" only, i.e. long-living stars with initial masses $m$ (in units of $M_{\odot}$ ) in the range $m_{1} \leq m \leq m_{\mathrm{RGT}}$, where $m_{1}=0.1$ (BGM and W94) or 0.15 (BCT) and $m_{\mathrm{RGT}}$ is the initial mass of a star at the red giant tip. $m_{\mathrm{RGT}}$ was taken from Bertelli et al. (1994) for the BCT data, and was approximated by the turn-off mass $m_{\mathrm{TO}}$, as given in Buzzoni (1989 and 1995), for the BGM data.

To obtain the SSP data per unit mass, we assumed $m$ in the range $m_{1} \leq m \leq 100 M_{\odot}$ and evaluated the remnant masses $m_{\mathrm{r}}(m)$ (in $M_{\odot}$ ) according to the rule: $m_{\mathrm{r}}(m)=1.4$ for $m \geq 8 M_{\odot}$ and $m_{\mathrm{r}}(m)=0.077 m+0.48$ for $m_{\mathrm{RGT}}<m<8 M_{\odot}$ (Renzini \& Ciotti 1993). The mass lost by stars during the first ascent along the red-giant branch was neglected in evaluating the mass of the luminous component.

BGM include metallicities up to $Z=0.1$ at the age of $15 \mathrm{Gyr}$, and BCT at the ages 1 to $13 \mathrm{Gyr}$; we shall use the BGM and BCT libraries at the ages just mentioned. W94 considers the ages 1.5 to $17 \mathrm{Gyr}$ for $Z \geq 0.01$, and 8 to $17 \mathrm{Gyr}$ for $Z<0.01$, as we shall do in the present paper when using the W94 library. It should be noted (see Fig. 2) that the time

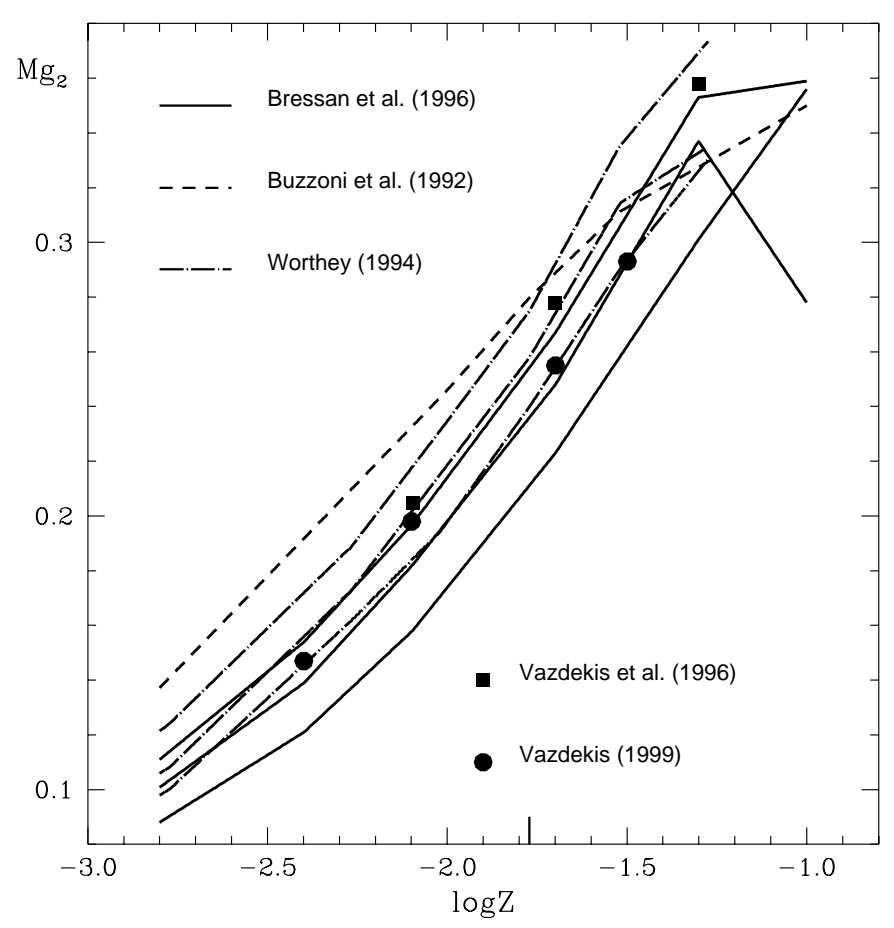

Fig. 2. SSP index $M g_{2}$ versus metal abundance $Z$, for the Salpeter's mass exponent $x=2.35$ : i) from Bressan et al. (1996) (solid curves) for the ages of 5, 9, and $13 \mathrm{Gyr}$ (from bottom to top); ii) from Buzzoni et al. (1992) for the age of $15 \mathrm{Gyr}$ (dashed curve); iii) from Worthey (1994, dotted-dashed curves) for the ages of 8, 12, and 17 Gyr (from bottom to top); iv) from Vazdekis et al. (1996, filled circles) and Vazdekis (1999, filled squares), for the age of $13 \mathrm{Gyr}$, for the sake of comparison. The thick mark on the abscissae indicates the solar metallicity.

behaviour of $\mathrm{Mg}_{2}$, as provided by W94, is monotonic at all $Z$, whereas $M g_{2}$ at $Z=0.1$, as given by BCT, has two minima at 1 and $7 \mathrm{Gyr}$, and two equal maxima at 5 and $13 \mathrm{Gyr}$.

The BCT, BGM, and W94 libraries adopt solar abundance ratios, whereas various authors found $[\mathrm{Mg} / \mathrm{Fe}]>0$ in giant ellipticals (Worthey et al. 1992; González 1993; Kuntschner 2000, TFWG1). As emphasized by TFWG1 (see also Weiss et al. 1995; Tantalo et al. 1998), that finding indicates an underabundance of iron (and related elements), rather than an overabundance of magnesium (and related elements), with respect to the solar values. Furthermore, TFWG1 apply previous results by Tripicco \& Bell (1995) to show that, for fixed $Z$ and with magnesium at the solar ratio, the underabundance of the iron-peak elements enhances the SSP index $M g_{2}$ with respect to the case with $[\mathrm{Mg} / \mathrm{Fe}]=0$, e.g. by about $9 \%$ for $Z=Z_{\odot}$, $[\mathrm{Mg} / \mathrm{Fe}]=0.3$, and an age of $12 \mathrm{Gyr}$ (see TFWG1, their Table 5).

A suitable set of SSP indices for non-solar abundance ratios, to be derived from new stellar evolutionary isocrones and new prescriptions like those by Tripicco \& Bell (1995), is as yet unavailable. Therefore, we were forced to use the index $M g_{2}$ as provided by the adopted SSP libraries. However, in the following Sect. 3.3 we shall discuss briefly the possible impact on our results by non-solar abundance ratios. 


\subsection{Model predictions}

The radial profile of index $M g_{2}$ depends on the age of stellar populations and model parameters, such as the concentration index $c$, the metal yield $p$, the exponent $n$ of the $R^{1 / n}$ law, and the anisotropy radius $r_{\mathrm{a}}$. Indeed, the $R^{1 / n}$ models can have an isotropic, or anisotropic, velocity dispersion, as explained in Appendix C. The anisotropic models used in this paper (the Osipkov-Merritt models) are specified by the anisotropy radius $r_{\mathrm{a}}\left(0<r_{\mathrm{a}}<+\infty\right)$; for them we adopt $r_{\mathrm{a}}=1$, since those with $r_{\mathrm{a}}<1$ are precluded by stability considerations (Ciotti \& Lanzoni 1997) and the models with $r_{\mathrm{a}}>1$ are superseded by those with $r_{\mathrm{a}}=+\infty$ (i.e. by isotropic models).

The effect of an age spread among the stellar populations is shown in Figs. 3 and 4 for $c=0.75$ and $p=Z_{\odot}$, and with reference to the isotropic $R^{1 / 4}$ model and the W94 library. The radial distance $R$ is given in units of the effective radius $R_{\mathrm{e}}(B)$, the radius of a centered circular aperture containing half of the total $B$ light. The initial age $\tau_{0}$ is $13 \mathrm{Gyr}$ for all the profiles. In Fig. 3a the final age $\tau_{\mathrm{w}}$, corresponding to the final $g_{\mathrm{w}}=0.005$, ranges from 11 to $2 \mathrm{Gyr}$, and the age spread between $\tau_{0}$ and $\tau_{\mathrm{w}}$ is that predicted by the Schmidt's law with $k=1$ (see Eqs. (6) to (9)). The $M g_{2}$ profile with no age spread ( $\left.\tau_{\mathrm{w}}=13 \mathrm{Gyr}\right)$ is also plotted for the sake of comparison.

Figure 3a shows that the radial profile of $M g_{2}$ is influenced appreciably by our adopted age spread only within $0.1 R_{\mathrm{e}}(B)$, where the observations of $M g_{2}$ in real ellipticals are usually disturbed by the seeing. In the region outside $0.1 R_{\mathrm{e}}(B)$, the age spread as formulated here has a negligible effect on the observed $\mathrm{Mg}_{2}$. Furthermore, the mean luminosity-weighted age within $R_{\mathrm{e}}(B) / 2$ is older than the age within $R_{\mathrm{e}}(B) / 8$ by $3 \%, 6 \%$, $14 \%$, and $27 \%$, when $\tau_{\mathrm{w}}=11,9,5$, and $2 \mathrm{Gyr}$, respectively. Therefore, an age increase by $25 \%$ from $R_{\mathrm{e}}(B) / 8$ out to the aperture radius $R_{\mathrm{e}}(B) / 2$, according to TFWG1, is verified only when $\tau_{\mathrm{w}}=2$ Gyr. However, further options, like those suggested by TFWG2 and Kodama \& Arimoto (1997 and 1998), are conceivable: they imply (see Sect. 2.3) a main episode of star formation, from $\tau_{0}=13 \mathrm{Gyr}$ to $\tau_{1}$ and involving the gas mass $1-g_{1}$, followed by a delayed minor episode occurring from $\tau_{2}$ to $\tau_{\mathrm{w}}$ and involving the gas mass $g_{1}-g_{\mathrm{w}}$.

Two of such alternatives are shown in Fig. 3b: they refer to $\tau_{1}=11 \mathrm{Gyr}, \tau_{2}=5 \mathrm{Gyr}, \tau_{\mathrm{w}}=4 \mathrm{Gyr}$, and $g_{1}=0.05$ or 0.1 . The mean luminosity-weighted age within $R_{\mathrm{e}}(B) / 2$ is older than the age within $R_{\mathrm{e}}(B) / 8$ by $27 \%$ and $38 \%$ when $g_{1}=0.05$ and 0.1 , respectively. Figure $3 \mathrm{~b}$ also displays the $M g_{2}$ profile at the age $\tau=\tau_{\mathrm{w}}=13 \mathrm{Gyr}$ with no age spread: the comparison between the two kinds of options reveals that $M g_{2}$ is affected appreciably only within $0.1 R_{\mathrm{e}}(B)$, as for the profiles in Fig. 3a. The mean ages $\left\langle\tau_{V}\right\rangle$ of the stars, weighted by the $V$ luminosity and as a function of the aperture radius $R$, are displayed in Fig. 4 for a model with $\tau_{\mathrm{w}}=11 \mathrm{Gyr}$ and a model with $\tau_{1}=11 \mathrm{Gyr}, \tau_{2}=5 \mathrm{Gyr}, \tau_{\mathrm{w}}=4 \mathrm{Gyr}$, and $g_{1}=0.05$.

In the following Figs. 5 to 7 we focus on the $M g_{2}$ profile at $R>0.1 R_{\mathrm{e}}(B)$ and, according to previous findings, we disregard any age spread by assuming $\tau_{\mathrm{w}}=\tau_{0}$. Figure 5 refers to the $M g_{2}$ profile for $c=0.75$ and the isotropic $R^{1 / 4}$ model; panel a shows the age-metallicity degeneracy of index $M g_{2}$ by using the W94 library: the profile with $\tau_{0}=17 \mathrm{Gyr}$ and $p=Z_{\odot}$

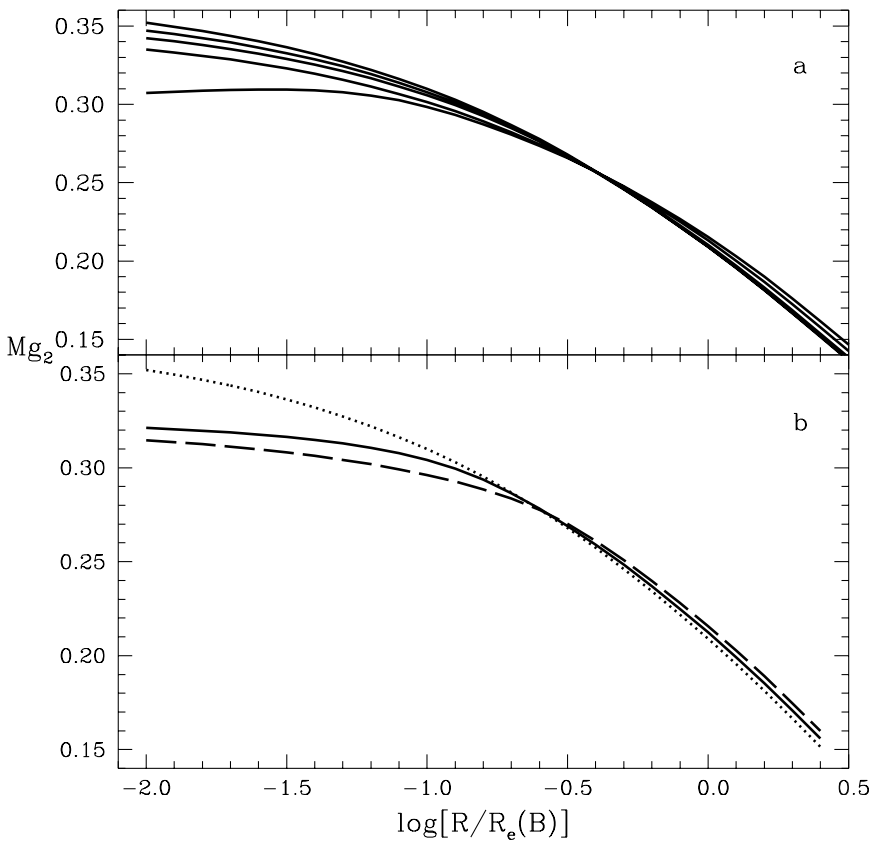

Fig. 3. The $M g_{2}$ profile from the Worthey (1994) library and for the isotropic $R^{1 / 4}$ model with an age spread among the stellar populations (see text). The projected radial distance $R$ is in units of the effective radius $R_{\mathrm{e}}(B)$, the radius of a centered circular aperture containing half of the total $B$ light. a) The initial age $\tau_{0}$ is $13 \mathrm{Gyr}$ for all the profiles and the final ages $\tau_{\mathrm{w}}$ are (from top to bottom) 13, 11, 9, 5 , and $2 \mathrm{Gyr}$, respectively. The profile with $\tau_{\mathrm{w}}=13 \mathrm{Gyr}$ has no age spread and is shown for the sake of comparison. b) Two profiles correspond to model ellipticals with the bulk of star formation occurring from 13 to 11 Gyr ago and a delayed final burst, 1 Gyr long starting from $5 \mathrm{Gyr}$ ago and involving the remaining fractional masses of 0.05 (solid curve) and 0.1 (dashed curve). The profile for a model at the age of $13 \mathrm{Gyr}$ and with no age spread is also plotted for the sake of comparison (dotted curve).

is matched by the profile with $\tau_{0}=8 \mathrm{Gyr}$ and $p=1.7 Z_{\odot}$, and similarly the profile with $\tau_{0}=8 \mathrm{Gyr}$ and $p=Z_{\odot}$ is matched by the profile with $\tau_{0}=17 \mathrm{Gyr}$ and $p=0.63 Z_{\odot}$. According to Eqs. (1) and (4), all the profiles in Fig. 5 correspond to the same gradient of $Z / p$. By fitting an observed $M g_{2}$ profile we can infer the underlying gradient of $Z / p$, but we cannot determine $p$ if the age is unknown.

The profiles in Fig. 5a should change for $[\mathrm{Mg} / \mathrm{Fe}]>$ 0, since $M g_{2}$ enhances when iron is below the solar value (Sect. 2.3). On the other hand, $[\mathrm{Mg} / \mathrm{Fe}]$ appears to be constant within each elliptical, although it varies from elliptical to elliptical (Worthey et al. 1992; Davies et al. 1993, TFWG1). However, for a fixed $[\mathrm{Mg} / \mathrm{Fe}]$ the enhancement of the SSP index $\mathrm{Mg}_{2}$ depends on $\mathrm{Z}$ and age. TFWG1 only report a $9 \%$ increase for $Z=Z_{\odot}$ and the age of $12 \mathrm{Gyr}$. If the fractional enhancement of $M g_{2}$ varied mildly with $Z$ and age, then the profiles in Fig. 5a would be shifted upwards with a minor change of their radial gradients.

Our adopted SSP libraries are compared in Figs. 5b-c by using the isotropic $R^{1 / 4}$ model with $c=0.75$ and $p=1.5 Z_{\odot}$. Figure $5 \mathrm{~b}$ shows the comparison between BGM and W94 at the age of 15 Gyr, and Fig. 5c presents the comparison between BCT and W94 at the age of 13 Gyr. The largest central 


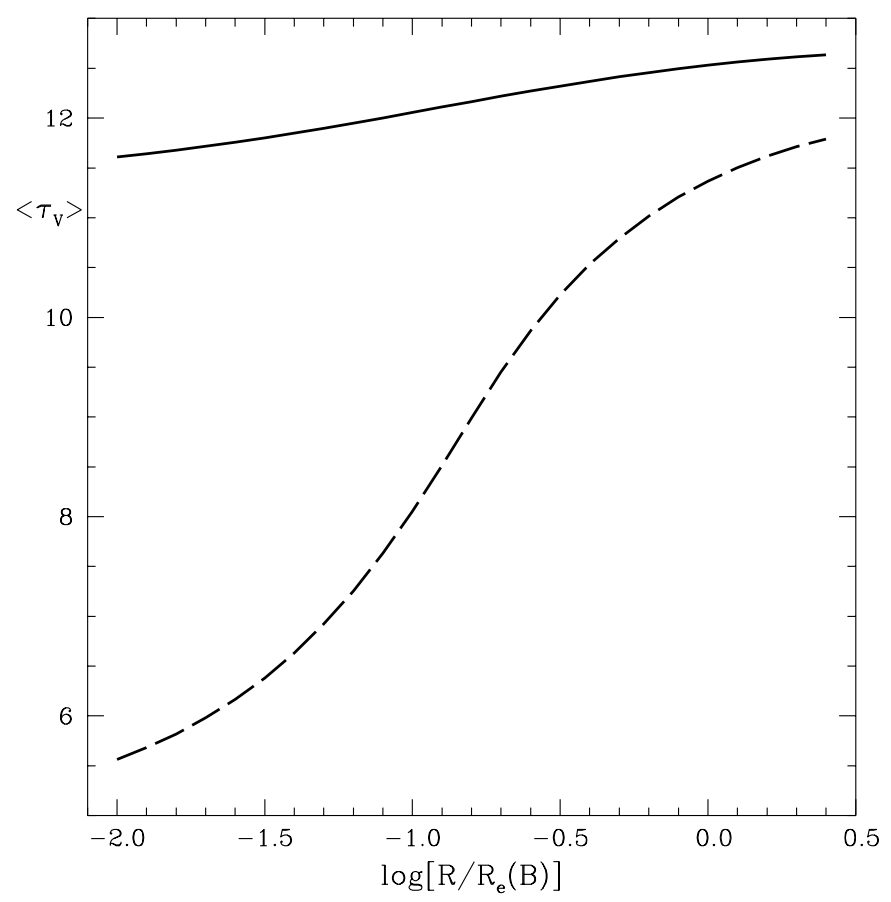

Fig. 4. Mean ages $\left\langle\tau_{V}>\right.$ (in Gyr), weighted by the $V$ luminosity and as a function of the aperture radius $R$, for two model ellipticals considered in Fig. 3b. In the first model (solid curve) all stars form from 13 to $11 \mathrm{Gyr}$ ago; in the second model (dashed curve) the fraction 0.95 of all stars forms from 13 to $11 \mathrm{Gyr}$ ago and the remaining fraction 0.05 between 5 and 4 Gyr ago. $R_{\mathrm{e}}(B)$ is as in Fig. 3 .

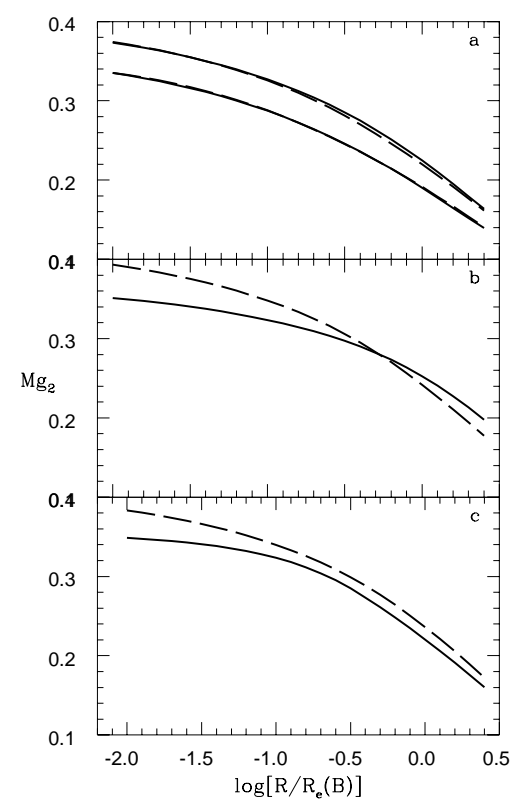

Fig. 5. $M g_{2}$ profiles, for the isotropic $R^{1 / 4}$ model, versus the projected radial distance $R$ in units of $R_{\mathrm{e}}(B)$, with $R_{\mathrm{e}}(B)$ as in Fig. 3 . a) Age-metallicity degeneracy by using the Worthey (1994) library: profiles for the age of $8 \mathrm{Gyr}$ (solid curves) with $p=Z_{\odot}$ and $1.7 Z_{\odot}$ (bottom to top), compared to profiles for the age of $17 \mathrm{Gyr}$ (dashed curves) with $p=0.63 Z_{\odot}$ and $Z_{\odot}$ (bottom to top). b) Profiles, for the age of $15 \mathrm{Gyr}$ and corresponding to $p=1.5 Z_{\odot}$, from the Buzzoni et al. (1992) library (solid curve) and Worthey (1994) library (dashed curve). c) Profiles, for the age of $13 \mathrm{Gyr}$ and corresponding to $p=$ $1.5 Z_{\odot}$, from the Bressan et al. (1996) library (solid curve) and Worthey (1994) library (dashed curve).

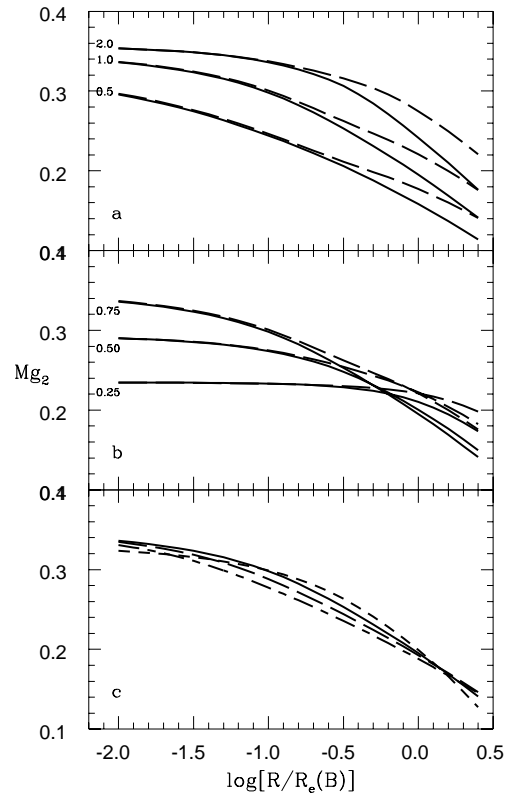

Fig. 6. a) Radial profiles of $M g_{2}$ versus $\log \left(R / R_{\mathrm{e}}\right)$ for the $R^{1 / 4}$-mass model, Concentration Models with $c=0.75$, and the labelled values of $p / Z_{\odot}$. The solid curves refer to the isotropic models and the dashed curves to the anisotropic models with $r_{\mathrm{a}}=1 . R_{\mathrm{e}}(B)$ is the effective radius in the $B$ band. b) As in a) but for Concentration Models with $p=Z_{\odot}$ and the labelled values of $c$. c) As in a) but for Concentration Models with $c=0.75, p=Z_{\odot}$, and isotropic $R^{1 / n}$-mass models with $n=2$ (short-dashed curve), 4 (solid curve), 6 (long-dashed curve), and 8 (short-dashed-long-dashed curve). The anisotropic $\left(r_{\mathrm{a}}=1\right) R^{1 / n}$ models, omitted for clarity reasons, behave like the isotropic ones.

values of $M g_{2}$ are those from the W94 library, owing to the extrapolation of the SSP data for $Z>0.053$ (see Fig. 2). The flattest profile is that from BGM, whereas the profiles from BCT and W94 have a comparable slope; such a behaviour is related to the slope $\mathrm{d} \mathrm{Mg}_{2} / \mathrm{d} \log Z$ of the SSP index $M g_{2}$ versus $Z$ (see Fig. 2).

We now use the BCT library, for the age of $13 \mathrm{Gyr}$, to show in Fig. 6 how the $M g_{2}$ profile depends on parameters $p, c, r_{\mathrm{a}}$, and $n$. Figure 6 a refers to fixed $c=0.75, r_{\mathrm{a}}=1$ or $+\infty, n=4$, and a variable $p$; Fig. $6 \mathrm{~b}$ to fixed $p=Z_{\odot}, r_{\mathrm{a}}=1$ or $+\infty, n=4$, and a variable $c$; and Fig. $6 \mathrm{c}$ to a variable $n$ with fixed $c=$ $0.75, p=Z_{\odot}$, and $r_{\mathrm{a}}=+\infty$. It is worth noting in panels a and $\mathrm{b}$ that the anisotropic models have $M g_{2}$ profiles flatter than those of the isotropic models.

Finally, Fig. 7 displays some profiles obtained with the consideration of a terminal wind removing the gas mass $g_{\mathrm{w}}=0.05$ from the sphere with $s_{1}\left(m_{\mathrm{w}}\right)=0.1$ (see Sect. 2.2). Here we use the isotropic $R^{1 / 4}$ model, the BCT library for the age of $13 \mathrm{Gyr}$, and the same metal yield $p=Z_{\odot}$ for all the profiles. One can now see that index $M g_{2}$ is weaker and its profile is flatter, especially in the central regions, than in the absence of a terminal wind. However, such an effect is appreciable only for $c \geq 0.5$, and is due mostly to $s_{1}\left(m_{\mathrm{W}}\right)>0$ rather than to $g_{\mathrm{w}}>0$. Indeed, the profiles with $s_{1}\left(m_{\mathrm{w}}\right)=0.1$ and $g_{\mathrm{w}}$ in the range 0 to 0.1 are almost identical with those in Fig. 7. 
Table 1. Parameters of Concentration Models matching the $M g_{2}$ profiles in 11 elliptical galaxies (from Davies et al. 1993) as displayed in Figs. 8 to 12. In the order, the columns list: the galaxy name, the $B$-band effective radius in arcsec (from Burstein et al. 1987), the concentration index, the metal yield, the cumulative mass (in units of the total stellar mass) of the stars with apocentres within the sphere with mass $m_{\mathrm{w}}$, the index of the $R^{1 / n}$-mass model, the anisotropy radius ( $+\infty$ for the isotropic models) in units of the effective radius $R_{\mathrm{e}}^{0}$ of the $R^{1 / n}$-mass model, the $B$-band effective radius of the best fitting model (in units of $R_{\mathrm{e}}^{0}$ ).

\begin{tabular}{lrllllll}
\hline \hline Name & $R_{\mathrm{e}}$ & $c$ & $p / Z_{\odot}$ & $s_{1}\left(m_{\mathrm{w}}\right)$ & $n$ & $r_{\mathrm{a}}$ & $R_{\mathrm{e}}(B)$ \\
\hline NGC 315 & 58.5 & 0.75 & 1.45 & 0.13 & 4 & 1.0 & 1.495 \\
NGC 741 & 52.1 & 0.75 & 1.25 & 0.13 & 4 & $+\infty$ & 1.728 \\
NGC 1600 & 47.5 & 0.80 & 2.2 & 0.13 & 8 & $+\infty$ & 2.547 \\
NGC 3379 & 35.2 & 0.667 & 1.4 & 0.08 & 4 & 1.0 & 1.488 \\
NGC 4261 & 38.6 & 0.95 & 1.7 & 0.0 & 4 & 1.0 & 1.526 \\
NGC 4278 & 32.9 & 0.667 & 1.4 & 0.08 & 4 & $+\infty$ & 1.725 \\
NGC 4374 & 54.6 & 0.667 & 1.55 & 0.08 & 5 & 1.0 & 1.723 \\
NGC 4472 & 104.0 & 0.6 & 2.2 & 0.0 & 6 & 1.0 & 1.855 \\
NGC 4486 & 104.0 & 0.95 & 2.0 & 0.0 & 6 & 2.0 & 2.038 \\
NGC 4636 & 101.7 & 0.75 & 1.1 & 0.0 & 4 & $+\infty$ & 1.735 \\
NGC 7626 & 37.8 & 0.8 & 1.65 & 0.0 & 7 & 1.0 & 2.234 \\
\hline
\end{tabular}

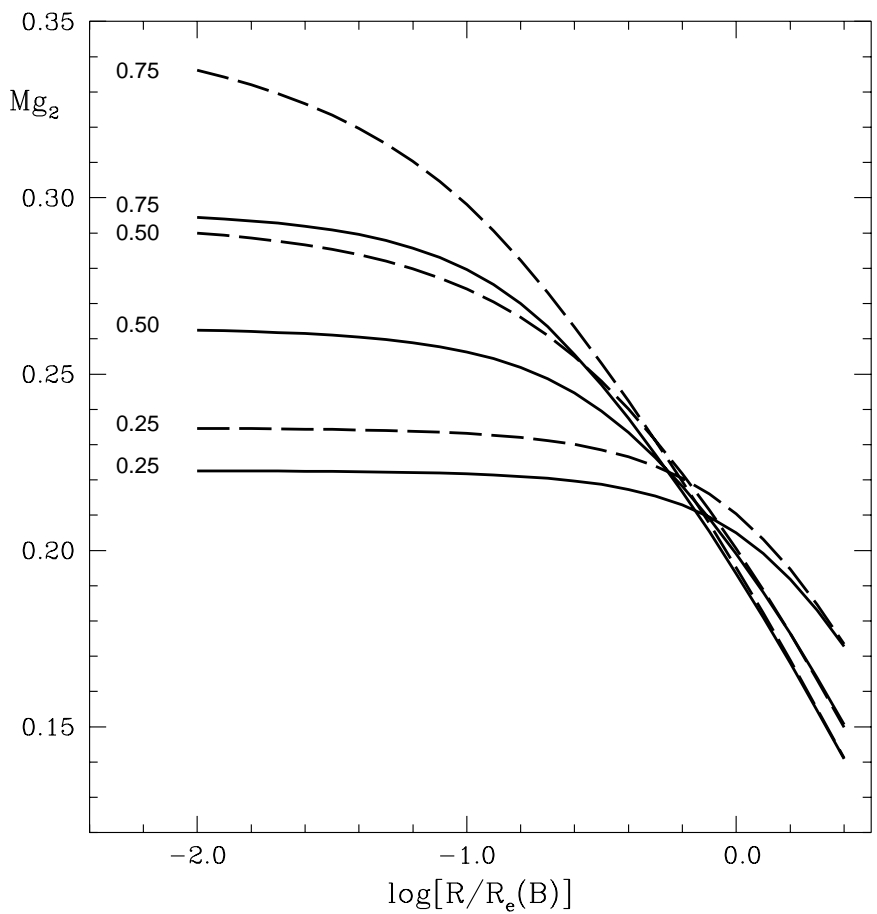

Fig. 7. $M g_{2}$ profiles for the isotropic $R^{1 / 4}$ model and with the consideration of a terminal wind removing the gas mass $g_{\mathrm{w}}=0.05$ from model ellipticals (see text for further details). The projected radial distance $R$ is in units of $R_{\mathrm{e}}(B)$, as in Fig. 3. All profiles are from the Bressan et al. (1996) library, for the age of $13 \mathrm{Gyr}$, and correspond to $p=Z_{\odot}$. For each value of the concentration index $c$ (labelling the curves), there are a profile with a terminal wind (solid curve) and a profile without a terminal wind (dashed curve).

\section{Comparison with observations}

The model predictions of Sect. 3.3 are here compared to the observations in a sample of 11 ellipticals. We shall use the BCT library for the age of 13 Gyr with complementary considerations of the BGM and W94 libraries and of an age spread among the stellar populations. Figures 8 to 12 display our best fitting profiles (after eye inspection) for the $M g_{2}$ profiles as measured by Davies et al. (1993, hereafter DSP) and tabulated by them along the intermediate galaxy axis. The effective radii from RC2 (de Vaucouleurs et al. 1976), adopted by DSP, are replaced here by the $B$-band radii $R_{\mathrm{e}}$ from Burstein et al. (1987). The crosses along the model curves correspond to the radius $\left(3^{\prime \prime}\right)$ of the seeing disk: observed points within two seeing disks are probably affected by seeing and should be excluded from the comparison. For each elliptical of the DSP sample, Table 1 lists the galaxy name, the observed $R_{\mathrm{e}}$ (in $\operatorname{arcsec})$, and parameters $c, p, n, r_{\mathrm{a}}$, and $R_{\mathrm{e}}(B)$ of our best model. If the inner points, affected by seeing, are included in the comparison, then some model profiles require the values of $s_{1}\left(m_{\mathrm{w}}\right)>0$ listed in Table 1 .

In fitting the $M g_{2}$ profile for NGC 741, we excluded the observed points at $\log R / R_{\mathrm{e}}=-0.55$ and -0.80 (see Fig. 10), which are about $0.03 \mathrm{mag}$ below the average values of the neighbouring points and cannot be matched by our models. Furthermore, the observed $M g_{2}$ profile for NGC 4486 (see Fig. 12) drops from $0.358 \mathrm{mag}$ at $\log R / R_{\mathrm{e}}=-1.5$ to 0.349 at $\log R / R_{\mathrm{e}}=-1.74$ and to $0.277 \mathrm{mag}$ at $\log R / R_{\mathrm{e}}=-2.32$, possibly denoting lower metallicities or younger ages near the centre. We did not try composite models to match such a behaviour and therefore we excluded the innermost two points.

Six Es, out of eleven, have shallow profiles within about $0.1 R_{\mathrm{e}}(B)$ followed by steep profiles up to $R_{\mathrm{e}}(B)$ : they cannot be fitted by isotropic or anisotropic CMs with $s_{1}\left(m_{\mathrm{w}}\right)=0$. The flat profiles within $0.1 R_{\mathrm{e}}(B)$ are affected by seeing and can be discarded; however, one can try to reproduce such profiles by CMs with $s_{1}\left(m_{\mathrm{w}}\right)>0$. For the ease of presentation, we adopted $s_{1}\left(m_{\mathrm{w}}\right)>0$ and $g_{\mathrm{w}}=0$ (see Sect. 3.3); however, the observed profiles can be fitted equally well by CMs with values of $s_{1}\left(m_{\mathrm{w}}\right)$ similar to those in Table 1 but with $0<g_{\mathrm{w}} \leq s_{1}\left(m_{\mathrm{w}}\right)$ and a small upward adjustment of $p$. The shallow profiles can also be reproduced by CMs with an age spread among the stellar populations, as discussed in Sect. 3.3 and shown in Fig. 8 for the $M g_{2}$ profile of NGC 3379.

The difference between the isotropic and anisotropic CMs, shown in Figs. 6a-b, is also illustrated in Figs. 8 and 11. NGC 3379 and NGC 4278 have $M g_{2}$ profiles almost 


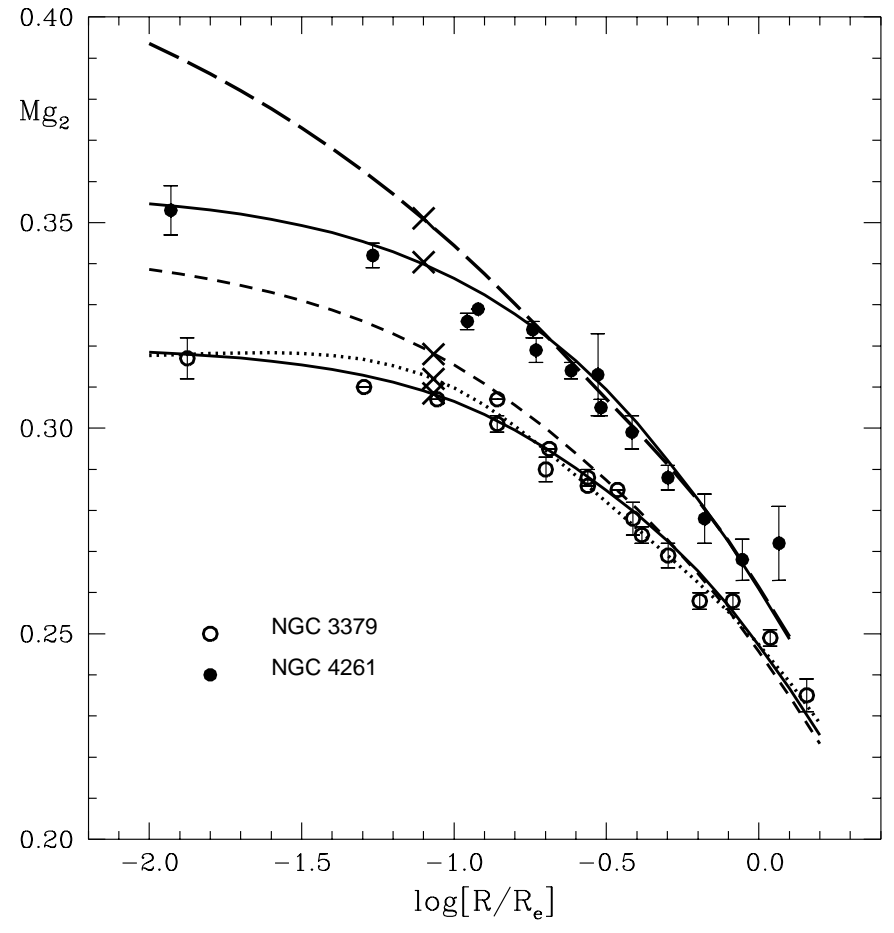

Fig. 8. Radial profiles of $M g_{2}$ versus $\log \left(R / R_{\mathrm{e}}\right)$, as measured by Davies et al. (1993) in the two indicated galaxies, compared to model profiles (solid curves) obtained from the Bressan et al. (1996) library and with the parameters for the Concentration Model and $R^{1 / n_{-}}$ mass model as specified in Table 1. Two further profiles are added for NGC 3379: i) a profile (short-dashed curve) from the Bressan et al. (1996) library for the age of $13 \mathrm{Gyr}$ and with parameters as specified in Table 1, except $s_{1}\left(m_{\mathrm{w}}\right)=0$ and $R_{\mathrm{e}}(B) / R_{\mathrm{e}}^{0}=1.504$; ii) a profile (dotted curve) from the Worthey (1994) library and with a star formation proceeding from 13 to $2 \mathrm{Gyr}$ (according to Eq. (6) with $k=1$ ), and with parameters as specified in Table 1 , except $s_{1}\left(m_{\mathrm{w}}\right)=0, p=1.2 Z_{\odot}$, and $R_{\mathrm{e}}(B) / R_{\mathrm{e}}^{0}=1.22$. An additional profile (dashed curve) is also shown for NGC 4261: it was computed from the Worthey (1994) library for the age of $13 \mathrm{Gyr}$ and with parameters as listed in Table 1, except $p=1.45 Z_{\odot}$ and $R_{\mathrm{e}}(B) / R_{\mathrm{e}}^{0}=1.445$. The radial distances $R$ are in units of $R_{\mathrm{e}}$ (second column in Table 1, unless otherwise indicated) for the observational points and in units of $R_{\mathrm{e}}(B)$ (last column in Table 1) for the model profiles. The crosses on the model profiles correspond to the radius $\left(3^{\prime \prime}\right)$ of the seeing disk.

overlapping each other up to about $0.2 R_{\mathrm{e}}(B)$ and diverging farther out: they are fitted by two CMs that differ only in the anisotropic radius $r_{\mathrm{a}}$. Moreover, we choose NGC 3379 to show in Fig. 8 the effect of $s_{1}\left(m_{\mathrm{w}}\right)>0$ : if one sets $s_{1}\left(m_{\mathrm{w}}\right)=0$, the remaining parameters in Table 1 being unaltered, the model profile coincides with that in Fig. 8 in the radial range from $0.1 R_{\mathrm{e}}(B)$ to $R_{\mathrm{e}}(B)$ while diverging upwards within $0.1 R_{\mathrm{e}}(B)$. If the inner profiles, observed in the afore-mentioned six Es, are flattened by seeing, then a fair fit to the true inner profiles could be provided by CMs with the same parameters listed in Table 1, except $s_{1}\left(m_{\mathrm{w}}\right)=0$.

The $M g_{2}$ profiles in five Es are fairly well matched, outside two seeing disks, by CMs with $s_{1}\left(m_{\mathrm{w}}\right)=0$; however, within two seeing disks, model values are below the observed values for NGC 4486 and NGC 7626, or barely reach them for NGC 4261, NGC 4472, and NGC 4636. The model fitting

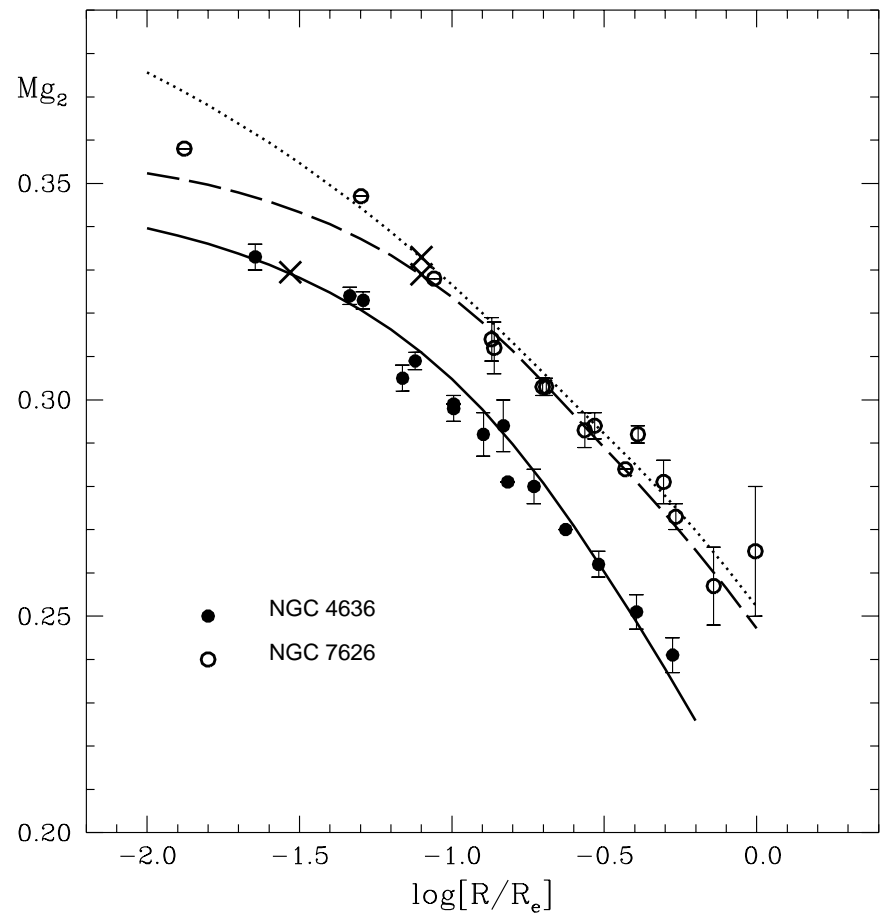

Fig. 9. As in Fig. 8 but with the solid curve for NGC 4636 and the dashed curve for NGC 7626, and with both curves from the Bressan et al. (1996) library for the age of $13 \mathrm{Gyr}$ (see Table 1). An additional profile (dotted curve) for NGC 7626 was derived from the Worthey (1994) library for the age of $13 \mathrm{Gyr}$ and with parameters as listed in Table 1, except $p=1.45 Z_{\odot}$ and $R_{\mathrm{e}}(B) / R_{\mathrm{e}}^{0}=1.974$.

would worsen if inner profiles were depressed by seeing and/or by an age effect (see Sect. 3.3). We also note that NGC 4261, with $M g_{2}=0.353$ near the centre and a steep $M g_{2}$ gradient between $0.1 R_{\mathrm{e}}(B)$ to $R_{\mathrm{e}}(B)$, requires $c=0.95$. Such a large concentration implies a very peaked metallicity distribution: about $98 \%$ of the mass is contained, according to Eq. (A.6), in the range $0.8 Z_{\mu}$ to $Z_{\mu}$ (see Appendix A). The same remark applies to NGC 4486. Furthermore, NGC 4486 (Fig. 12) and NGC 7626 (Fig. 9) have $M g_{2} \simeq 0.36$ near the centre. Such a large $M g_{2}$ value is obtainable hardly from the SSP data in the BCT library, which reaches the largest value $M g_{2}=0.359$ at $Z=0.1$ (see Fig. 2). To a lesser extent, the same problem also holds for NGC 4261 (Fig. 8).

For NGC 4636 and NGC 4472 we could not find profiles better than those shown in Figs. 9 and 10, respectively, by using the W94 library; on the contrary, alternative profiles from the W94 library are possible for NGC 4261, NGC 7626, and NGC 4486, as shown in Figs. 8, 9, and 12, respectively. The model profiles from W94 correspond to the age of $13 \mathrm{Gyr}$, all other parameters being those listed in Table 1 for BCT, except $p$ and $R_{\mathrm{e}}(B)$, which are given in the figure's captions. The ability of the W94 library to reach, or exceed, the $M g_{2}$ values observed within two seeing disks is due to the extrapolation of the SSP index $M g_{2}$ beyond $Z=0.053$, as already mentioned in Sect. 3.3 and shown in Figs. 5b-c. However, the central profiles of the afore-mentioned five Es could not be fitted by the W94 library, if they were further depressed by an age effect, 


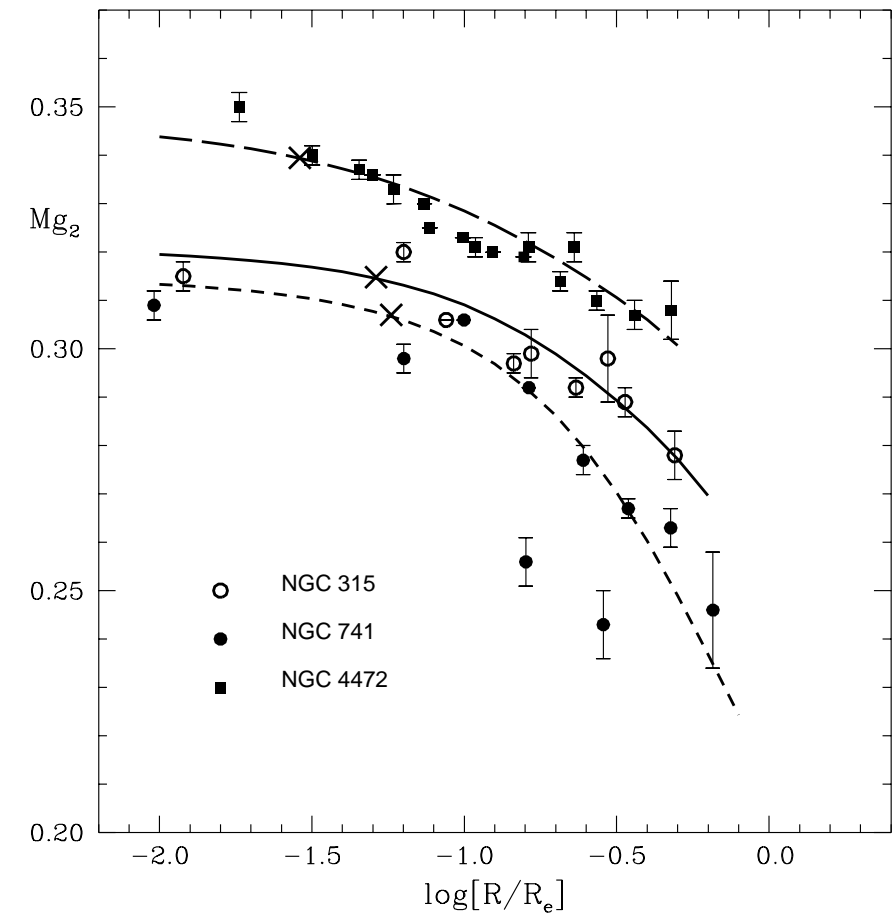

Fig. 10. As in Fig. 8 but with the solid curve for NGC 315, the shortdashed curve for NGC 741, and the long-dashed curve for NGC 4472. All curves are from the Bressan et al. (1996) library for the age of $13 \mathrm{Gyr}$ (see Table 1).

unless the metallicity gradient is steeper than that predicted by the $\mathrm{CM}$

The farthest points observed by DSP correspond to radial distances $\gtrsim R_{\mathrm{e}}(B)$ in five Es (NGC 3379, NGC 4261, NGC 4278, NGC 4374, and NGC 7626) and are in the range $\simeq 0.5 R_{\mathrm{e}}(B)$ to $\simeq 0.7 R_{\mathrm{e}}(B)$ in the remaining six Es. Taking into account ratios $R_{\mathrm{e}}(B) / R_{\mathrm{e}}^{0}$ in Table 1 , one concludes that the fittings shown in Figs. 8 to 12 refer to regions enclosing in projection about $63 \%$ to $72 \%$ of the masses of the five afore-mentioned Es, and about $48 \%$ to $62 \%$ for the remaining Es, except NGC 315 for which the mass fraction is $42 \%$ only.

The $R^{1 / n}$-mass model, with $n>4$, fits the $M g_{2}$ profiles of five Es of the DSP sample better than the $R^{1 / 4}$-mass model. Parameter $n$ in Table 1 refers to the BCT library for the age of $13 \mathrm{Gyr}$, and was found by varying parameters $n, c$, and $p$, in an attempt at matching the observed points outside $0.1 R_{\mathrm{e}}(B)$. However, $n$ also depends on the adopted library: indeed, by using the $R^{1 / 4}$-mass model with the W94 library, and disregarding the observed points within two seeing disks, a fair fit can be found for all the Es with $n>4$ as listed in Table 1, except for NGC 4472.

Parameter $n$ of the $R^{1 / n}$-mass model, as obtained here from the $M g_{2}$ profile, can be compared with the shape parameter $n$ of the Sersic's law, as derived by some authors from the luminosity profile (for the Sersic's profiles, see Trujillo et al. 2001). Four Es in Table 1 (NGC 4374, NGC 4472, NGC 4486, and NGC 4636) are in common with the samples studied by Caon et al. (1993) in the $B$ band and by Graham \& Colless (1997) in the $V$ band: their Sersic parameters are significantly different in the quoted references and they also differ from the

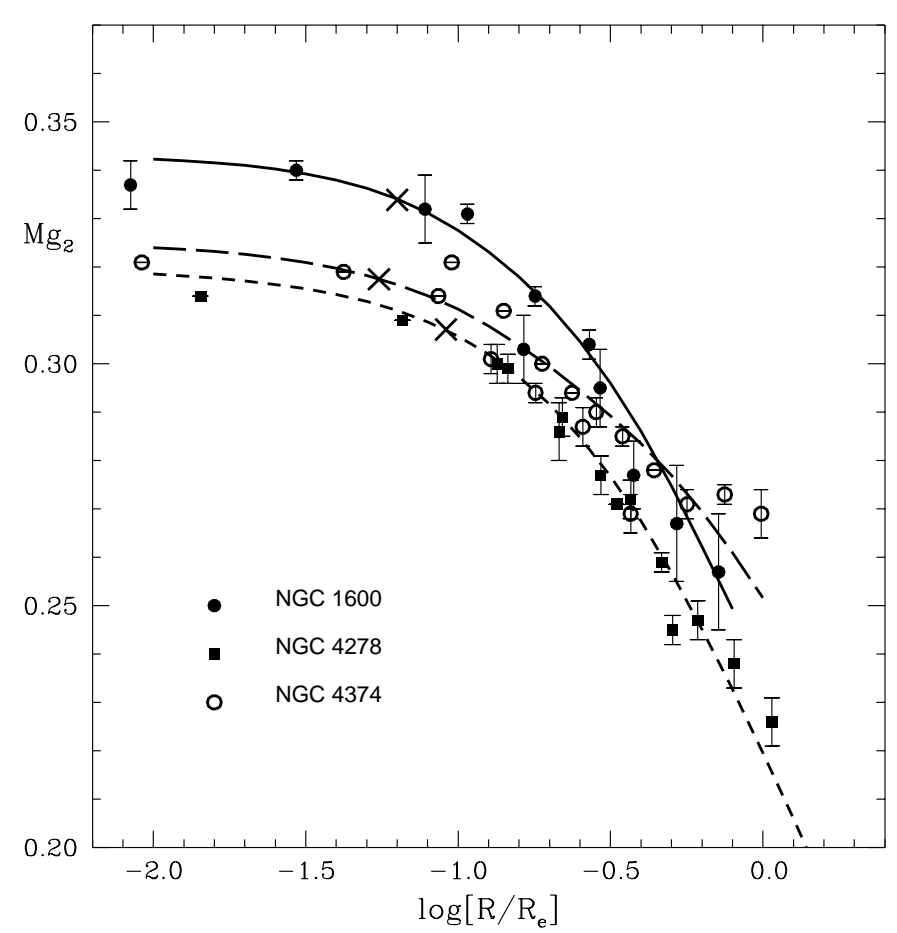

Fig. 11. As in Fig. 8 but with the solid curve for NGC 1600, the short-dashed curve for NGC 4278, and the long-dashed curve for NGC 4374. All curves are from the Bressan et al. (1996) library for the age of $13 \mathrm{Gyr}$ (see Table 1).

values of $n$ given in Table 1. Caon et al. (1993) also report $n=7.68$ for NGC 4261, against our $n=4$. It is unknown to what extent the disagreement between the quoted authors can be ascribed to their adopted photometric bands. The CM, when used in conjunction with the $R^{1 / n}$-mass model, gives $B$-bandluminosity profiles that agree within 0.1 mag with the Sersic's profiles, from about $0.1 R_{\mathrm{e}}$ up to $4 R_{\mathrm{e}}$ (at least). On the other hand, some decoupling between the $M g_{2}$ and the luminosity profiles could be expected on the ground that the SSP photometric properties, unlike the SSP index $M g_{2}$, are affected only slightly (at fixed $Z$ ) by the non-solar ratio $[\mathrm{Mg} / \mathrm{Fe}]$ (TFWG1; Tantalo et al. 1998).

We conclude this section by noting that the $M g_{2}$ profiles, in some Es of the DSP sample, can be fitted with model profiles from the BGM library with practically the same values of $p, c$, and $n$ as given in Table 1, but with a different value of $r_{\mathrm{a}}$. The observed profiles matched by the anisotropic models when using the BCT library are also fitted by the isotropic models when using the BGM library.

\section{Discussion and conclusions}

The Concentration Model is admittedly an oversimplified version of more elaborate dissipative models of galaxy formation. However, Sects. 3 and 4 show that the Concentration Model, when used in conjunction with the $R^{1 / n}$ model and the Singleburst Stellar Populations index $M g_{2}$ from available libraries, leads to non-generic predictions that compare fairly well, outside the inner two seeing disks, with detailed observations in a sample of 11 ellipticals. Such a specific comparison was the 


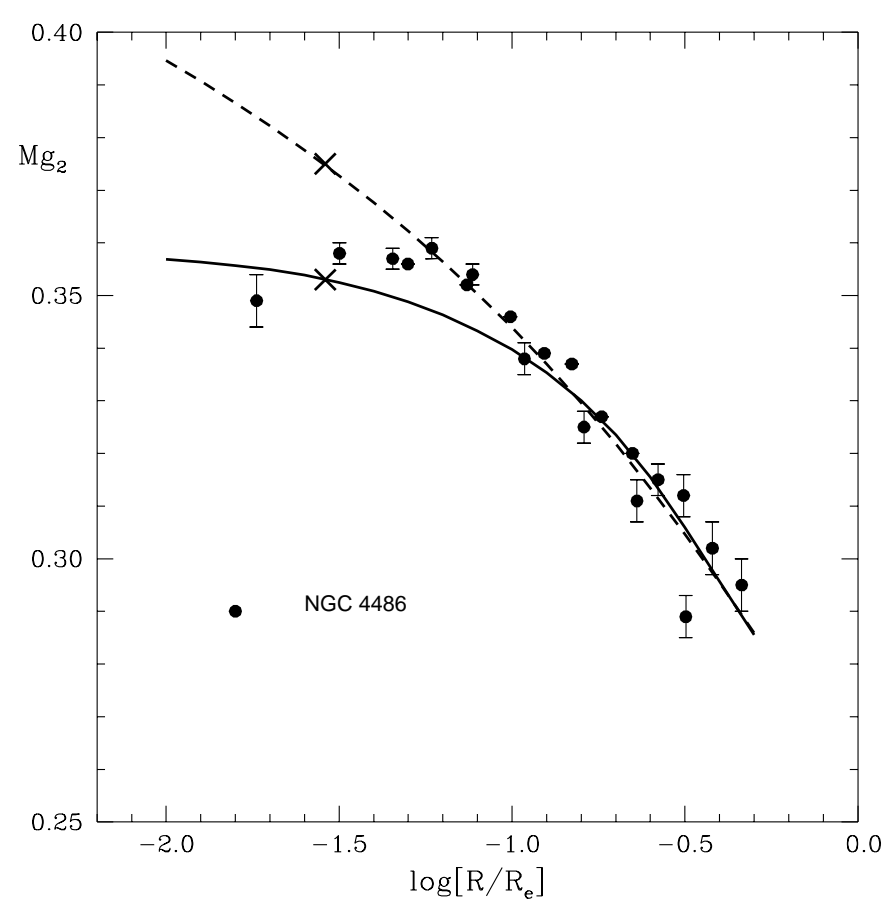

Fig. 12. As in Fig. 8 but for NGC 4486 with model profiles from: i) the Bressan et al. (1996) library for the age of $13 \mathrm{Gyr}$ and parameters as in Table 1 (solid curve), ii) from the Worthey (1994) library for the age of $13 \mathrm{Gyr}$ and parameters as in Table 1 , except $p=1.7 Z_{\odot}$ and $R_{\mathrm{e}}(B) / R_{\mathrm{e}}^{0}=1.881$ (dashed curve).

main goal of the present paper, although it has to be considered as a preliminary test to further investigations.

Our results depend obviously on the mass distribution of the $R^{1 / n}$ model, which is only a rough approximation to real elliptical galaxies, since it is a spherical model derived from the luminosity profile by assuming a constant mass-to-light ratio. Moreover, our results also depend on the dynamical structure, as shown by the $M g_{2}$ profiles obtained with isotropic and anisotropic Osipkov-Merritt models. Further computations are needed to assess to what extent the integrated $\mathrm{Mg}_{2}$ is affected by the mass and dynamical structure of alternative models of elliptical galaxies.

The age spread, among the stellar populations of individual ellipticals, was considered in a very simple way, following the suggestion by TFWG1 and Kodama \& Arimoto (1997 and 1998). A qualitative agreement can be found with the age gradients inferred by TFWG1 in a sample of 39 ellipticals, but the issue would require a more suitable index and more detailed comparisons than the few experiments we have done with index $M g_{2}$. Moreover, our age spread produces $M g_{2}$ profiles that are flat in the innermost regions. The Concentration Model with a terminal wind offers an alternative possibility of obtaining such profiles, at the cost of introducing the additional parameters $m_{\mathrm{w}}$ and $g_{\mathrm{w}}$.

The free parameters $m_{\mathrm{w}}$ and $g_{\mathrm{w}}$ are related, presumably, to the depth of the potential well, the degree of dissipation, and the star-formation and supernovae rates (see Pagel 1997). Hydrodynamical computations, like those performed by various authors (Larson 1976; Carlberg 1984; Theis et al. 1992), are required to obtain an acceptable relation between $g_{\mathrm{w}}$ and $s_{1}\left(m_{\mathrm{w}}\right)$. The specific values for $g_{\mathrm{w}}$ and $s_{1}\left(m_{\mathrm{w}}\right)$, as adopted in Sect. 3, were chosen arbitrarily for illustrative purpose only.

The comparison with observations, as discussed in Sect. 4, suffers from the seeing effects: the observed $M g_{2}$ profiles, within two seeing disks, are uncertain, so we cannot say what kind of Concentration Model (with or without an age spread and with or without a terminal wind) is the more appropriate one for each individual elliptical galaxy. A further uncertainty comes from the poorly known effects of non-solar abundance ratios on index $M g_{2}$. If the enhancement of the SSP index $M g_{2}$, for a variable $Z$ with a fixed $[\mathrm{Mg} / \mathrm{Fe}]>0$, is like that induced by a fixed age increase for a variable $Z$, then our model profiles could be considered as fair approximations to the observed profiles. In such a case, as in the case of the age/metallicity degeneracy considered in Sect. 3.3, we could infer the underlying gradient of $Z$ without determining $Z$ in absolute units. Obviously, this issue could only be assessed when an adequate SSP library, covering a reasonable range of nonsolar $[\mathrm{Mg} / \mathrm{Fe}]$ ratios, were available.

Besides the above uncertainties, there are some degeneracies, illustrated in Sect. 3.3, among some parameters that are needed in our approach. We find that the slope of steep profiles, outside (say) $0.1 R_{\mathrm{e}}(B)$, is determined chiefly by the concentration index $c$, and that the specific values of index $M g_{2}$ along the profiles are controlled mainly by the metal yield $p$ (for a fixed age). However, a heavy degeneracy among model parameters persists when dealing with shallow $M g_{2}$ profiles within $R_{\mathrm{e}}(B)$.

We reiterate that the comparison with observations, discussed in Sects. 3.3 and 4, is only a preliminary test of our approach and is by no means conclusive, since it does not include colour indices and spectral indices other than $\mathrm{Mg}_{2}$. Moreover, the comparison is affected by a major uncertainty stemming from the SSP libraries we have used, which are admittedly unsatisfactory to study the spectro-photometric properties of giant ellipticals.

We summarize the main results of the paper as follows:

- i) the Concentration Model by Lynden-Bell (1975) has been slightly modified to include a terminal wind and an age spread among the stellar populations, and then adapted to a mass model of elliptical galaxies, derived by deprojection from the luminosity profile obeying the $R^{1 / n}$ law;

- ii) by using the calibration of the $M g_{2}$ index as a function of metallicity from Single-burst Stellar Populations models, the $M g_{2}$ profiles in 11 individual elliptical galaxies have been matched by some specific model profiles;

- iii) the Concentration Model appears to reproduce reasonably well the observed $M g_{2}$, at least from $0.1 R_{\mathrm{e}}(B)$ up to $R_{\mathrm{e}}(B)$, and requires concentration indices $c \geq 0.5$ and metal yields $p>Z_{\odot}$. A terminal wind, with additional parameters $m_{\mathrm{w}}$ and $g_{\mathrm{w}}$, is a possibility for elliptical galaxies with flat $M g_{2}$ profiles in the innermost regions. However, scaling relations, derived from physical processes governing the galaxy formation, are needed to link together concentration index, metal yield, $m_{\mathrm{w}}$, and $g_{\mathrm{w}}$, and thus to place the Concentration Model on a firmer basis;

- iv) the comparison with observations is affected by major uncertainties due to the seeing, that influences the observed 
$M g_{2}$ profile within about $0.1 R_{\mathrm{e}}(B)$, and to the adopted SSP libraries, that do not include the effects of non-solar abundance ratios on index $\mathrm{Mg}_{2}$;

- v) owing to the seeing effects, we could not determine what kind of Concentration Models (with or without a terminal wind, with or without an age spread) were best suited to the $M g_{2}$ profiles of six ellipticals, out of eleven ellipticals studied in the present paper;

- vi) besides the well-known age/metallicity degeneracy of index $\mathrm{Mg}_{2}$, there are some degeneracies of model parameters when the computed $M g_{2}$ profiles are compared to the observed ones. The concentration index $c$ seems affected less than the remaining model parameters by such degeneracies when dealing with steep $M g_{2}$ profiles within $R_{\mathrm{e}}(B)$.

Acknowledgements. We would like to thank an anonimous referee for her/his valuable suggestions that helped to improve the content and presentation of this paper.

\section{Appendix A: General solutions}

Cumulative mass $\sigma\left(m, Z_{\mathrm{a}}\right)$ and metallicity-distribution function $M D F$ are derived here starting from ansatz (5). To ease the presentation, we introduce the abbreviations $\mu=s_{1}(m)$ and $\widetilde{g}=$ $\left(g-g_{\mathrm{w}}\right) /\left(1-g_{\mathrm{w}}\right)$ with subscripts "a" or "w" when $m=m_{\mathrm{a}}$ or $m_{\mathrm{w}}$, and $g=g_{\mathrm{a}}$ or $g_{\mathrm{w}}$, respectively. We shall also use quantity $\widetilde{\mu}=\left(\mu-\mu_{\mathrm{w}}\right) /\left(1-\mu_{\mathrm{w}}\right)$ together with subscripts "a" or "w" when $\mu=\mu_{\mathrm{a}}$ or $\mu_{\mathrm{w}}$, respectively.

Therefore, Eq. (5) can now be rewritten as

$\mu_{\mathrm{a}}=\mu_{\mathrm{w}}+\left(1-\mu_{\mathrm{w}}\right) \widetilde{g}_{\mathrm{a}}^{c}$.

Furthermore, gas metallicity $Z_{\mathrm{a}}=Z_{0}-p \ln g_{\mathrm{a}}$ will be denoted by $Z_{\mu}$ when $g_{\mathrm{a}}=g$ or $m_{\mathrm{a}}=m$, and by $Z_{\mathrm{w}}$ when $g_{\mathrm{a}}=g_{\mathrm{w}}$ or $m_{\mathrm{a}}=m_{\mathrm{w}}$.

\section{A.1. Cumulative mass $\sigma\left(m, Z_{\mathrm{a}}\right)$}

We refer to a "fixed" $m$ in a galaxy after $t_{\mathrm{w}}$, and first consider the alternative $m \geq m_{\mathrm{w}}$. The stars with apocentres inside $m$ have metallicities from $Z_{0}$ to $Z_{\mathrm{w}}$ : those with $Z_{\mathrm{a}}=Z_{0}$ to $Z_{\mu}$ formed before $t_{\mathrm{w}}$ when $m_{\mathrm{a}} \geq m$, and those with $Z_{\mathrm{a}}=Z_{\mu}$ to $Z_{\mathrm{w}}$ formed when $m_{\mathrm{a}} \leq m$. We discuss the cases $m_{\mathrm{a}} \leq m$ and $m_{\mathrm{a}} \geq m$ in the following items I) and II), respectively, and defer the alternative $m \leq m_{\mathrm{w}}$ to item III).

I) Case $m_{\mathrm{a}} \leq m$

Mass $\sigma\left(m, Z_{\mathrm{a}}\right)$ follows from the obvious condition $M_{\mathrm{s}}\left[\sigma\left(m, Z_{\mathrm{a}}\right)+s_{2}(m)+s_{3}(m)\right]+M_{0} g_{\mathrm{a}}=M_{0}$, that is

$\sigma\left(m, Z_{\mathrm{a}}\right)=\mu-\widetilde{g}_{\mathrm{a}}$

where $\widetilde{g}_{\mathrm{a}}=\widetilde{g}\left(Z_{\mathrm{a}}\right)$. If $m_{\mathrm{a}}=m_{\mathrm{w}}$, then $\widetilde{g}\left(Z_{\mathrm{w}}\right)=0$ and $\sigma\left(m, Z_{\mathrm{w}}\right)$ equals the final value $s_{1}(m) \equiv \mu$.

II) Case $m_{\mathrm{a}} \geq m$

Mass $\sigma\left(m, Z_{\mathrm{a}}\right)$ is solution of equation

$M_{\mathrm{s}} \frac{\mathrm{d} \sigma\left(m, Z_{\mathrm{a}}\right)}{\mathrm{d} t_{\mathrm{a}}}=-M_{0} \frac{\sigma\left(m, Z_{\mathrm{a}}\right)}{\sigma\left(m_{\mathrm{a}}, Z_{\mathrm{a}}\right)} \frac{\mathrm{d} g_{\mathrm{a}}}{\mathrm{d} t_{\mathrm{a}}}$ where $Z_{\mathrm{a}}$ and $m_{\mathrm{a}}$ depend on $t_{\mathrm{a}}$ through $g_{\mathrm{a}}=g\left(t_{\mathrm{a}}\right)$. Eq. (A.3) also reads

$\frac{\mathrm{d} \sigma\left(m, Z_{\mathrm{a}}\right)}{\sigma\left(m, Z_{\mathrm{a}}\right)}=-\frac{\mathrm{d} \widetilde{g}_{\mathrm{a}}}{\mu_{\mathrm{w}}+\left(1-\mu_{\mathrm{w}}\right) \widetilde{g}_{\mathrm{a}}^{c}-\widetilde{g}_{\mathrm{a}}}$.

After integration from the fixed point $m_{\mathrm{a}}=m$ to $m_{\mathrm{a}}>m$, one has

$\frac{\sigma\left(m, Z_{\mathrm{a}}\right)}{\sigma\left(m, Z_{\mu}\right)}=F\left(\mu, Z_{\mathrm{a}}\right)$

where $\sigma\left(m, Z_{\mu}\right)=\mu-\widetilde{\mu}^{1 / c}$ from Eq. (A.2) at $m_{\mathrm{a}}=m$ and from Eq. (A.1) at $\mu_{\mathrm{a}}=\mu$. Moreover, one has

$F\left(\mu, Z_{\mathrm{a}}\right)=\exp \left[-\int_{\widetilde{g}(\mu)}^{\widetilde{g}\left(Z_{\mathrm{a}}\right)} \frac{\mathrm{d} x}{\mu_{\mathrm{w}}+\left(1-\mu_{\mathrm{w}}\right) x^{c}-x}\right]$

where $\widetilde{g}(\mu)=\widetilde{\mu}^{1 / c}$ from Eq. (A.1) at $\mu_{\mathrm{a}}=\mu$, and with $F\left(\mu, Z_{0}\right)=0$ and $F\left(\mu, Z_{\mu}\right)=1$. One gets $F\left(\mu, Z_{\mathrm{a}}\right)=\{[1-$ $\left.\left.\widetilde{g}^{1-c}\left(Z_{\mathrm{a}}\right)\right] /\left[1-\widetilde{g}^{1-c}(\mu)\right]\right\}^{1 /(1-c)}$ when $m_{\mathrm{w}}=0$. In the general case $m_{\mathrm{w}}>0$, we obtain $F\left(\mu, Z_{\mathrm{a}}\right)$ numerically from Eq. (A.6). In addition to that, we also used in the paper some analytical solutions of Eq. (A.6) which can be found for special values of index $c(1 / 3,1 / 2,2 / 3$, and $3 / 4$; see Appendix B).

III) Case $m \leq m_{\mathrm{w}}$

The stars with $Z_{\mathrm{a}}=Z_{0}$ to $Z_{\mathrm{w}}$ all formed when $m_{\mathrm{a}}$ varied from 1 to $m_{\mathrm{w}}$ : for them $\sigma\left(m, Z_{\mathrm{w}}\right)$ coincides with mass $s_{1}(m) \equiv \mu$ of the final distribution. By integrating Eq. (A.4) from the fixed point $m_{\mathrm{a}}=m_{\mathrm{w}}$ to $m_{\mathrm{a}}>m_{\mathrm{w}}$ one gets

$\frac{\sigma\left(m, Z_{\mathrm{a}}\right)}{\sigma\left(m, Z_{\mathrm{w}}\right)}=F\left(\mu_{\mathrm{w}}, Z_{\mathrm{a}}\right)$

where $F\left(\mu_{\mathrm{w}}, Z_{\mathrm{a}}\right)$ is as in Eq. (A.6) with $\widetilde{g}(\mu)$ replaced by $\widetilde{g}\left(\mu_{\mathrm{w}}\right)=0$ from Eq. (A.1) at $\mu_{\mathrm{a}}=\mu_{\mathrm{w}}$. Function $F\left(\mu_{\mathrm{w}}, Z_{\mathrm{a}}\right)$ is obtained as described in item II.

\section{A.2. Metallicity distribution}

The stars with apocentres on the surface corresponding to $m \geq$ $m_{\mathrm{w}}$ completed their formation when $m_{\mathrm{a}}=m$. Their fractional mass distribution, in the metallicity range $Z_{\mathrm{a}}$ to $Z_{\mathrm{a}}+\mathrm{d} Z_{\mathrm{a}}$, follows from Eq. (A.5) as $\operatorname{MDF}\left(\mu, Z_{\mathrm{a}}\right) \mathrm{d} Z_{\mathrm{a}}$, where

$\operatorname{MDF}\left(\mu, Z_{\mathrm{a}}\right)=\frac{\partial F\left(\mu, Z_{\mathrm{a}}\right)}{\partial Z_{\mathrm{a}}}$

with $Z_{\mathrm{a}}=Z_{0}$ to $Z_{\mu}$. On the other hand, the stars with apocentres on the surface corresponding to $m \leq m_{\mathrm{w}}$ completed their formation when $m_{\mathrm{a}}=m_{\mathrm{w}}$, and one gets from Eq. (A.7) that

$\operatorname{MDF}\left(\mu_{\mathrm{w}}, Z_{\mathrm{a}}\right)=\frac{\partial F\left(\mu_{\mathrm{w}}, Z_{\mathrm{a}}\right)}{\partial Z_{\mathrm{a}}}$

with $Z_{\mathrm{a}}=Z_{0}$ to $Z_{\mathrm{w}}$ and $\operatorname{MDF}\left(\mu_{\mathrm{w}}, Z_{\mathrm{a}}\right)$ independent of $m$. Equations (A.8) and (A.9) are the main results of CM that are actually used here in conjunction with the $R^{1 / n}$ model (for technical details see Appendix C). 


\section{Appendix B: Particular solutions}

Solutions (A.6) for $c=1 / 3,1 / 2,2 / 3,3 / 4$, and $\mu_{\mathrm{w}}>0$ can be written as

$F\left(\mu, Z_{\mathrm{a}}\right)=\frac{P(y)}{P(x)}$

where $x$ is a power of $\widetilde{\mu}, y$ a power of $\widetilde{g}\left(Z_{\mathrm{a}}\right)$, and $P(q)$ (with $q=x$ or $q=y)$ is as specified below. Solutions $F\left(\mu_{\mathrm{w}}, Z_{\mathrm{a}}\right)$ of Eq. (A.7) differ from solutions (A.6) in that $\widetilde{\mu}_{\mathrm{a}}$ becomes $\widetilde{\mu}_{\mathrm{w}}=$ 0 and, therefore, $P(x)$ in Eq. (B.1) is replaced by $P(0)$.

I) Case c $=1 / 3$

One has $x=\widetilde{\mu}, y=\widetilde{g}^{1 / 3}\left(Z_{\mathrm{a}}\right)$, and $P(q)$ depending on $\mu$ as detailed in the following items i) to iii):

i) $\mu>0.25$

$P(q)=(1-q)^{\alpha}\left(q^{2}+q+\mu_{\mathrm{w}}\right)^{\beta} \exp \left[-\gamma \operatorname{atan}\left(\frac{2 q+1}{k}\right)\right]$

where $\alpha=3 /\left(2+\mu_{\mathrm{w}}\right), \beta=\left[3\left(1+\mu_{\mathrm{w}}\right)\right] /\left[2\left(2+\mu_{\mathrm{w}}\right)\right], \gamma=[3(1-$ $\left.\left.\mu_{\mathrm{w}}\right)\right] /\left[k\left(2+\mu_{\mathrm{w}}\right)\right]$, and $k=\left(4 \mu_{\mathrm{w}}-1\right)^{1 / 2}$.

ii) $\mu=0.25$

$P(q)=(1-q)^{\alpha}(q+0.5)^{\beta} \exp \left(\frac{\gamma}{2 q+1}\right)$

where $\alpha=3 /\left(2+\mu_{\mathrm{w}}\right), \beta=\left[3\left(1+\mu_{\mathrm{w}}\right)\right] /\left[\left(2+\mu_{\mathrm{w}}\right)\right]$, and $\gamma=$ $\left[3\left(1-\mu_{\mathrm{w}}\right)\right] /\left[\left(2+\mu_{\mathrm{w}}\right)\right]$.

iii) $\mu<0.25$

$P(q)=(1-q)^{\alpha}\left(q+r_{1}\right)^{\beta}\left(q+r_{2}\right)^{\gamma}$

where $\alpha=3 /\left(2+\mu_{\mathrm{w}}\right), \beta=\left[6 \mu_{\mathrm{w}}-3(1-k)\right] /[k(3-k)], \gamma=$ $\left[3(1+k)-6 \mu_{\mathrm{w}}\right] /[k(3+k)], r_{1}=(1-k) / 2, r_{2}=(1+k) / 2$, and $k=\left(1-4 \mu_{\mathrm{w}}\right)^{1 / 2}$.

II) Case $c=1 / 2$

One has $x=\widetilde{\mu}, y=\widetilde{g}^{1 / 2}\left(Z_{\mathrm{a}}\right)$, and

$P(q)=(1-q)^{\alpha}\left(\mu_{\mathrm{w}}+q\right)^{2 \alpha}$

with $\alpha=2 /\left(1+\mu_{\mathrm{w}}\right)$.

III) Case $c=2 / 3$

By setting $x=\widetilde{\mu}^{1 / 2}$ and $y=\widetilde{g}^{1 / 3}\left(Z_{\mathrm{a}}\right)$ one obtains

$P(q)=(1-q)^{\alpha}\left(q^{2}+\mu_{\mathrm{w}} q+\mu_{\mathrm{w}}\right)^{\beta} \exp \left[\gamma \operatorname{atan}\left(\frac{2 q+\mu_{\mathrm{w}}}{k}\right)\right]$

where $\alpha=3 /\left(2+\mu_{\mathrm{w}}\right), \beta=\alpha \mu_{\mathrm{w}}, \gamma=2 \beta\left(1-\mu_{\mathrm{w}}\right) / k$, and $k=$ $\left(4 \mu_{\mathrm{w}}-\mu_{\mathrm{w}}^{2}\right)^{1 / 2}$.

IV) Case c $=3 / 4$

By setting $x=\widetilde{\mu}^{1 / 3}$ and $y=\widetilde{g}^{1 / 4}\left(Z_{\mathrm{a}}\right)$ one has

$P(q)=(1-q)^{\alpha}(q-r)^{\beta}(q+b q+d)^{\delta} \exp \left[-\gamma \operatorname{atan}\left(\frac{2 q+b}{k}\right)\right]$

where $b=\mu_{\mathrm{w}}+r, d=\mu_{\mathrm{w}}+b r$, and $k=\left(4 d-b^{2}\right)^{1 / 2} \cdot r$ is a real root of a cubic equation and reads as $r=h-\mu_{\mathrm{w}} / 3$, where $h=\left(-a_{1}+a_{2}\right)^{1 / 3}-\left(a_{1}+a_{2}\right)^{1 / 3}$ with $a_{1}=\mu_{\mathrm{w}}\left(2 \mu_{\mathrm{w}}^{2}-9 \mu_{\mathrm{w}}+27\right) / 54$ and $a_{2}=\mu_{\mathrm{w}}\left(3 \mu_{\mathrm{w}}^{2}-14 \mu_{\mathrm{w}}+27\right) / 54$. One also has

$\alpha=4-\beta-2 \delta$

$\beta=-\frac{4 r^{3}}{(1-r)(r+b r+d)}$

$\delta=2 \frac{4(2 b-r)-\beta(1-r)(r+b+1)}{b+d+1}$

$\gamma=2 \frac{4(b-r)-\beta(1-r)-\delta(b+2)}{k}$.

\section{Appendix C: The $R^{1 / n}$ model}

In this appendix the CM is adapted to the Sersic's $R^{1 / n}$ model of a spherical galaxy. Mass $M_{\mathrm{s}}$ and effective radius $R_{\mathrm{e}}^{0}$ (of the $R^{1 / n}$-mass model) are adopted as units of mass and length, respectively. The spatial radial distance will be indicated by $r$ and the projected radial distance by $R$.

The $R^{1 / n}$ model can be isotropic or anisotropic according to whether, at any location $r$, one has $\overline{v_{\mathrm{r}}^{2}}=\overline{v_{\mathrm{t}}^{2}}$, or $\overline{v_{\mathrm{r}}^{2}}>\overline{v_{\mathrm{t}}^{2}}$, respectively, where $\overline{v_{\mathrm{r}}^{2}}$ and $\overline{v_{\mathrm{t}}^{2}}$ are the dispersions of the squared radial $v_{\mathrm{r}}$ and transverse $v_{\mathrm{t}}$ velocities, respectively. Besides the isotropic models, we shall only consider a particular kind of anisotropic models (the so-called Osipkov-Merritt models) that have the same mass structure of the isotropic models with the same $n, M_{\mathrm{s}}$, and $R_{\mathrm{e}}^{0}$ but a different dynamical structure, i.e. a different velocity distribution. The Osipkov-Merritt models (see below) are specified by the anisotropy radius $r_{\mathrm{a}}\left(0<r_{\mathrm{a}}<\right.$ $+\infty)$ and reduce to the corresponding isotropic models in the limit $r_{\mathrm{a}}=+\infty$.

Gravitational potential $\Phi(r)$ and binding energy $\mathcal{E}$, both in physical units, will be replaced by the positive dimensionless potential $\psi(r)=\Phi(r) / \Phi_{0}$, where $\Phi_{0}=\Phi(0)$, and by the dimensionless energy $\varepsilon=-\mathcal{E} / \Phi_{0}$, respectively. One has $\varepsilon=\psi(r)-v^{2} / 2$ ( $v$ being the modulus of the spatial velocity) with $0 \leq \psi(r) \leq 1$ and $0 \leq \varepsilon \leq 1$.

The distribution function $f(\varepsilon)$ for the isotropic models, and $f(Q)$ for the anisotropic ones, are computed as described in the literature (Osipkov 1979; Binney 1982; Merritt 1985; Binney \& Tremaine 1987 for $n=4$; Ciotti 1991; Ciotti \& Lanzoni 1997 for the general case). In the anisotropic models (the so-called Osipkov-Merritt models), the distribution function $f(Q)$ depends on the dimensionless (i.e. in units of $-\Phi_{0}$ ) variable $Q=\varepsilon-|J|^{2} /\left(2 r_{\mathrm{a}}^{2}\right)$, where $J$ is the angular momentum and $r_{\mathrm{a}}$ the anisotropy radius, with $f(Q)=0$ for $Q<0$.

\section{C.1. Isotropic $R^{1 / n}$ models}

The mass coordinate $m$ in Sect. 2 is replaced by $M(r)$, the total mass inside the sphere of radius $r$. Therefore, we define $r_{m}$ by $M\left(r_{m}\right)=m$ and likewise $r_{\mathrm{a}}$ and $r_{\mathrm{w}}$ when $m=m_{\mathrm{a}}$ and $m_{\mathrm{w}}$, respectively. We also write $s_{1}\left(r_{m}\right)=s_{1}(m)=\mu$ and likewise $s_{1}\left(r_{\mathrm{a}}\right)=\mu_{\mathrm{a}}$ and $s_{1}\left(r_{\mathrm{w}}\right)=\mu_{\mathrm{w}}$.

At location $r, \varepsilon$ varies from 0 to $\psi(r)$ and $|J|=r v|\sin \vartheta|$ $(\vartheta$ being the angle between vectors $\boldsymbol{v}$ and $\boldsymbol{r}$ ) from 0 to $J_{r}(\varepsilon)=r v$, where $v=\{2[\psi(r)-\varepsilon]\}^{1 / 2}$. The apocentric distance 
$r_{\text {ap }}=r_{\text {ap }}(\varepsilon, J)$, solution of equation $\left\{2\left[\psi\left(r_{\text {ap }}\right)-\varepsilon\right]\right\}^{1 / 2} r_{\text {ap }}=J$, may be smaller, or larger, than $r_{\mathrm{w}}$. If $r_{\mathrm{ap}}>r_{\mathrm{w}}$, the differential distribution of metallicities, for fixed $\varepsilon$ and $J$, is then given by $\operatorname{MDF}\left(\mu_{\mathrm{ap}}, Z_{\mathrm{a}}\right)$ of Eq. (A.8), where $\mu_{\text {ap }}=s_{1}\left(r_{\mathrm{ap}}\right)$ and $Z_{\mathrm{a}}$ varies from $Z_{0}$ to $Z_{\text {ap }}=Z_{0}-p \ln g_{\text {ap }}$ with $g_{\text {ap }}=g_{\mathrm{w}}+\left(1-g_{\mathrm{w}}\right) \tilde{\mu}_{\mathrm{ap}}^{1 / c}$ and $\widetilde{\mu}_{\text {ap }}=\left(\mu_{\text {ap }}-\mu_{\mathrm{w}}\right) /\left(1-\mu_{\mathrm{w}}\right)$. If $r_{\mathrm{ap}}<r_{\mathrm{w}}$, then $\operatorname{MDF}\left(\mu_{\mathrm{w}}, Z_{\mathrm{a}}\right)$ is given by Eq. (A.9) with $Z_{\mathrm{a}}$ ranging from $Z_{0}$ to $Z_{\mathrm{w}}$.

We now consider a $Z$-dependent function $I(Z)$ and define $\widetilde{I}(\varepsilon, J)$ as the value (per unit mass) of $I(Z)$ integrated over all stars with fixed $\varepsilon$ and $J$. If $r_{\text {ap }}>r_{\mathrm{w}}$, one has

$\widetilde{I}(\varepsilon, J)=\int_{Z_{0}}^{Z_{\text {ap }}} \operatorname{MDF}\left(\mu_{\mathrm{ap}}, Z_{\mathrm{a}}\right) I\left(Z_{\mathrm{a}}\right) \mathrm{d} Z_{\mathrm{a}}$.

If $r_{\text {ap }}<r_{\mathrm{w}}$, then $\widetilde{I}(\varepsilon, J)$ follows from (C.1) with $Z_{\text {ap }}$ replaced by $Z_{\mathrm{w}}$ and $\operatorname{MDF}\left(\mu_{\mathrm{ap}}, Z\right)$ by $\operatorname{MDF}\left(\mu_{\mathrm{w}}, Z\right)$. Usually, one deals with a quantity $I(Z, \tau)$ that depends on metallicity $Z$ and age $\tau$. Equation (C.1) includes the particular case of a fixed age $\tau$, irrespective of $Z$, and also applies to the case of a one-to-one correspondence $\tau(Z)$, as in Eq. (9), by replacing $I(Z)$ with $I[Z, \tau(Z)]$.

We denote by $\mathcal{J}(r)$ the integrated value of $I(Z)$ due to all stars at location $r$. The contribution to $\mathcal{J}(r)$ from the stars with energies $\varepsilon$ to $\varepsilon+\mathrm{d} \varepsilon$ and momenta $J$ to $J+\mathrm{d} J$ is

$2 \pi \widetilde{I}(\epsilon, J) \frac{f(\varepsilon) J}{r\left[J_{r}^{2}(\varepsilon)-J^{2}\right]^{1 / 2}} \mathrm{~d} \varepsilon \mathrm{d} J$

hence

$\mathcal{J}(r)=4 \pi \int_{0}^{\psi(r)} f(\varepsilon) D(\varepsilon, r) \mathrm{d} \varepsilon$

where

$D(\varepsilon, r)=\frac{1}{r} \int_{0}^{J_{r}(\varepsilon)} \widetilde{I}(\epsilon, J) \frac{J}{\left[J_{r}^{2}(\varepsilon)-J^{2}\right]^{1 / 2}} \mathrm{~d} J$.

The integrated values $\mathcal{J}_{\mathrm{pr}}(R)$, along the line-of-sight at $R$, and $\mathcal{J}_{\text {ap }}(R)$, within a circular concentric aperture of radius $R$, follow as

$$
\begin{aligned}
\mathcal{J}_{\mathrm{pr}}(R)= & 8 \pi \int_{0}^{\psi(R)} f(\varepsilon) \\
& \times\left[\int_{R}^{r(\varepsilon)} D(\epsilon, r) \frac{r}{\left[r^{2}-R^{2}\right]^{1 / 2}} \mathrm{~d} r\right] \mathrm{d} \varepsilon
\end{aligned}
$$

and

$$
\begin{aligned}
\mathcal{J}_{\text {ap }}(R) & =16 \pi^{2} \int_{0}^{1} f(\varepsilon) \int_{0}^{r(\varepsilon)} D(\epsilon, r) r^{2} \mathrm{~d} r \mathrm{~d} \varepsilon \\
& -16 \pi^{2} \int_{0}^{\psi(R)} f(\varepsilon) \int_{R}^{r(\varepsilon)} D(\epsilon, r)\left(r^{2}-R^{2}\right)^{1 / 2} r \mathrm{~d} r \mathrm{~d} \varepsilon
\end{aligned}
$$

where $r(\varepsilon)$ is solution of $\psi[r(\varepsilon)]=\varepsilon$. If $I(Z)=1$, then Eq. (C.2) gives the local mass density $\varrho(r)$, Eq. (C.4) the mass density $\varrho_{\mathrm{pr}}(R)$ along the line-of-sight at $R$, and Eq. (C.5) the mass density $\varrho_{\text {ap }}(R)$ within the aperture radius $R$. Functions $\varrho_{\mathrm{pr}}(R)$ and $\varrho_{\text {ap }}(R)$ are available in elementary forms for the $R^{1 / n}$ model (see Young 1976 for $n=4$ ) and can be used to check the accuracy of numerical computations.

The integrated indices $\mathrm{Mg}_{2, \mathrm{pr}}(R)$ (along the line-of-sight at $R$ ) and $\operatorname{Mg}_{2, \text { ap }}(R)$ (within the centered aperture of radius $R$ ) are computed from published values of the synthetic SSP in$\operatorname{dex} \operatorname{Mg}_{2}(Z)$ (in mag), where $Z$ is the SSP metallicity. We write $F_{1}(Z)=F_{2}(Z) 10^{-0.4 \mathrm{Mg}_{2}(Z)}$, where $F_{1}(Z)$ is the SSP flux in the index passband and $F_{2}(Z)$ is the SSP-pseudocontinuum flux in the same passband. Both $F_{1}(Z)$ and $F_{2}(Z)$ refer to the unit mass. We make the approximation $F_{2}(Z)=a L_{V}(Z)$ (see Greggio 1997), where $L_{V}(Z)$ is the SSP luminosity in the $V$ band (and per unit mass) and factor $a$ is independent of $Z$. We then obtain

$\operatorname{Mg}_{2, \mathrm{pr}}(R)=-2.5 \log \frac{\mathcal{J}_{\mathrm{pr}}^{(1)}}{\mathcal{J}_{\mathrm{pr}}^{(2)}}$

and

$\operatorname{Mg}_{2, \mathrm{ap}}(R)=-2.5 \log \frac{\mathcal{J}_{\text {ap }}^{(1)}}{\mathcal{J}_{\text {ap }}^{(2)}}$

where $\mathcal{J}_{\mathrm{pr}}^{(1)}(R)$ and $\mathcal{J}_{\mathrm{pr}}^{(2)}(R)$ follow from Eq. (C.4) with $I(Z)=$ $F_{1}(Z)$ and $F_{2}(Z)$, respectively, and $\mathcal{J}_{\text {ap }}^{(1)}(R)$ and $\mathcal{J}_{\text {ap }}^{(2)}(R)$ from Eq. (C.5) with $I(Z)=F_{1}(Z)$ and $F_{2}(Z)$, respectively.

\section{C.2. Anisotropic $R^{1 / n}$ models}

At location $r$ one has $v=v(r, \vartheta)=\{2[\psi(r)-Q]\}^{1 / 2}[1+$ $\left.r^{2} \sin ^{2} \vartheta / r_{\mathrm{a}}^{2}\right]^{-1 / 2}$, where $Q$ varies from 0 to $\psi(r)$. Therefore, $|J|=$ $r v(r, \vartheta)|\sin \vartheta|$ varies from 0 to $J_{r}(Q)=v(r, \pi / 2) r$. The apocentric distance $r_{\text {ap }}=r_{\text {ap }}(Q, J)$ is solution of equation $v\left(r_{\text {ap }}, \pi / 2\right) r_{\text {ap }}=$ $J$ and $\widetilde{I}(\varepsilon, J)$ in Eq. (C.1) now becomes $\widetilde{I}(Q, J)$. Quantities $\mathcal{J}(r), \mathcal{J}_{\text {pr }}(R), \mathcal{J}_{\text {ap }}(R), \operatorname{Mg}_{2, \mathrm{pr}}(R)$, and $\operatorname{Mg}_{2, \text { ap }}(R)$ are given by Eqs. (C.2) and (C.4) to (C.7), respectively, when $\varepsilon$ is replaced by $\mathrm{Q}, r(\varepsilon)$ by $r(Q)$ \{where $r(Q)$ is solution of $\psi[r(Q)]=Q\}$, and $D(\varepsilon, r)$ by

$$
\begin{aligned}
D(Q, r)= & \frac{1}{r\left(1+r^{2} / r_{\mathrm{a}}^{2}\right)^{1 / 2}} \\
& \times \int_{0}^{J_{r}(Q)} \widetilde{I}(Q, J) \frac{J}{\left[J_{r}^{2}(Q)-J^{2}\right]^{1 / 2}} \mathrm{~d} J .
\end{aligned}
$$

\section{References}

Arimoto, N., \& Yoshii, Y. 1987, A\&A, 173, 23

Baugh, C. M., Cole, S., \& Frenk, C. 1996, MNRAS, 283, 1361

Bertelli, G., Bressan, A., Chiosi, C., et al. 1994, A\&AS, 106, 275

Binney, J. 1982, MNRAS, 200, 952

Binney, J., \& Tremaine, S. 1987, Galactic Dynamics (Princeton: Princeton University Press)

Bower, R. G., Lucey, J. R., \& Ellis, R. S. 1992, MNRAS, 254, 601

Bressan, A., Chiosi, C., \& Fagotto, F. 1994, ApJS, 94, 63

Bressan, A., Chiosi, C., \& Tantalo, R. 1996, A\&A, 311, 425 (BCT)

Burstein, D., Davies, R. L., Dressler, A., et al. 1987, ApJS, 64, 601

Burstein, D., Faber, S. M., Gaskell, C. M., et al. 1984, ApJ, 287, 586

Buzzoni, A. 1989, ApJS, 71, 817

Buzzoni, A. 1995, ApJS, 98, 69

Buzzoni, A., Gariboldi, G., \& Mantegazza, L. 1992, AJ, 103, 1814 (BGM)

Caon, N., Capaccioli, M., \& D’Onofrio, M. 1993, MNRAS, 265, 1013

Carlberg, R. G. 1984, ApJ, 286, 403

Carollo, C. M., Danziger, I. J., \& Buson, L. 1993, MNRAS, 265, 553 
Charlot, S., Worthey, G., \& Bressan, A. 1996, ApJ, 457, 625

Ciotti, L. 1991, A\&A, 249, 99

Ciotti, L., \& Lanzoni, B. 1997, A\&A, 321, 724

Cole, S., Aragon-Salamanca, A., Frenk, C. S., et al. 1994, MNRAS, 271,781

Davies, R. L., Sadler, E. M., \& Peletier, R. F. 1993, MNRAS, 262, 650 (DSP)

de Vaucouleurs, G. 1948, Ann. d'Astrophys., 11, 267

de Vaucouleurs, G., de Vaucouleurs, A., \& Corwin, H. G. 1976, Second Reference Catalogue of Bright Galaxies (Austin: University of Texas Press) (RC2)

Eggen, O. J., Lynden-Bell, D., \& Sandage, A. 1962, ApJ, 136, 748

Franx, M., \& Illingworth, G. 1990, ApJ, 359, L41

Franx, M., Illingworth, G., \& Heckman, T. 1989, AJ, 98, 538

González, J. J. 1993, Ph.D. Thesis, Univ. of California, Santa Cruz

Graham, A., \& Colless, M. 1997, MNRAS, 287, 221

Greggio, L. 1997, MNRAS, 285, 151

Jørgensen, I. 1999, MNRAS, 306, 607

Kauffmann, G. 1996, MNRAS, 281, 475

Kauffmann, G., \& Charlot, S. 1998, MNRAS, 297, 123

Kodama, T., \& Arimoto, N. 1997, A\&A, 320, 41

Kodama, T., \& Arimoto, N. 1998, MNRAS, 300, 193

Kuntschner, H. 2000, MNRAS, 315, 184

Larson, R. B. 1974, MNRAS, 169, 229

Larson, R. B. 1976, MNRAS, 176, 31

Lynden-Bell, D. 1975, Vistas in Astr., 19, 299 (LB)

Martinelli, A. 1998, A\&A, 335, 847

Matteucci, F., \& Tornambé, A. 1987, A\&A, 185, 51

Merritt, D. 1985, AJ, 90, 1027

Osipkov, L. P. 1979, Pis'ma Astron. Zh., 5, 77
Pagel, B. E. J. 1997, Nucleosynthesis and Chemical Evolution of Galaxies (Cambridge: Cambridge University Press)

Pagel, B. E. J., \& Patchett, B. E. 1975, MNRAS, 172, 13

Peletier, R. F., Davies, R. L., Illingworth, G. D., et al. 1990, AJ, 100, 1091

Renzini, A., \& Ciotti, L. 1993, ApJ, 416, L49

Schmidt, M. 1959, ApJ, 129, 243

Sersic, J.-L. 1968, Atlas de Galaxias Australes, Observatorio Astronomico, Cordoba

Tantalo, R., Chiosi, C., Bressan, A., et al. 1996, A\&A, 311, 361

Tantalo, R., Chiosi, C., Bressan, A., et al. 1998, A\&A, 333, 409

Terlevich, A. I., \& Forbes, D. A. 2002, MNRAS, 330, 547

Theis, Ch., Burkert, A., \& Hensler, G. 1992, A\&A, 265, 465

Trager, S. C., Faber, S. M., Worthey, G., et al. 2000a, AJ, 119, 1645 (TFWG1)

Trager, S. C., Faber, S. M., Worthey, G., et al. 2000b, AJ, 120, 165 (TFWG2)

Trager, S. C., Worthey, G., Faber, S. M., et al. 1998, ApJS, 116, 1

Tripicco, M. J., \& Bell, R. A. 1995, AJ, 110, 3035

Trujillo, I., Graham, A. W., \& Caon, N. 2001, MNRAS, 326, 869

Vazdekis, A. 1999, ApJ, 513, 225

Vazdekis, A., Casuso, E., Peletier, R. F., et al. 1996, ApJS, 106, 307

Vazdekis, A., Kuntschner, H., Davies, R. L., et al. 2001, ApJ, 551, L127

Visvanathan, N., \& Sandage, A. R. 1977, ApJ, 216, 214

Weiss, A., Peletier, R. F., \& Matteucci, F. 1995, A\&A, 296, 73

Worthey, G. 1994, ApJS, 95, 107 (W94)

Worthey, G., Faber, S. M., \& González, J. J. 1992, ApJ, 398, 69

Worthey, G., Faber, S. M., González, J. J., et al. 1994, ApJS, 94, 687

Young, P. J. 1976, AJ, 81, 807 